\title{
Compulsive sexual behavior, religiosity, and spirituality: A systematic review
}

\section{Journal of Behavioral Addictions}

10 (2021) 4, 854-878

DOI:

$10.1556 / 2006.2021 .00084$

(c) 2021 The Author(s)

\section{TODD L. JENNINGS ${ }^{1}$ (), TAYLER LYNG ${ }^{2}$ (0), NEIL GLEASON ${ }^{3}$ (1), ITOR FINOTELLI $^{4} \odot$ and ELI COLEMAN ${ }^{4 *} \odot$}

\author{
${ }^{1}$ Department of Psychology, University of Nevada, Las Vegas, United States \\ ${ }^{2}$ Department of Psychology, Minnesota State University, Mankato, United States \\ ${ }^{3}$ Department of Psychology, University of Washington, United States \\ ${ }^{4}$ The Institute for Sexual and Gender Health, University of Minnesota Medical School, University of \\ Minnesota, United States
}

Received: May 12, 2021 • Accepted: November 22, 2021

\section{REVIEW ARTICLE}

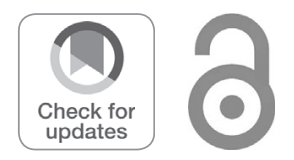

* Corresponding author. E-mail: dreli@umn.edu

\begin{abstract}
Background and aims: In recent years, increasing attention has been given to the relationship between compulsive sexual behavior (CSB), religiosity, and spirituality. This review summarizes research examining the relationship CSB has with religiosity and spirituality, clarifying how these constructs inform the assessment and treatment of this syndrome. Methods: The present paper reviews research published through August 1, 2021, using the Preferred Reporting Items for Systematic Reviews and Meta-analyses (PRISMA) guidelines. Only studies providing quantitative analyses were included. Results: This review identified 46 articles, subsuming 59 studies, analyzing the relationship between CSB and religiosity or spirituality. Most studies used cross-sectional designs with samples primarily composed of heterosexual White men and women. Generally, the studies found small to moderate positive relationships between religiosity and CSB. Studies considering the mediating or moderating role of moral incongruence identified stronger, indirect relationships between religiosity and problematic pornography use (PPU), a manifestation of CSB. Few studies examined the association between spirituality and $\mathrm{CSB}$, but those that did either reported negative relationships between indicators of spiritual well-being and CSB or positive relationships between CSB and aspects of spiritual struggles. Discussion and conclusions: Although research examining CSB and religiosity has flourished, such growth is hampered by cross-sectional samples lacking in diversity. Moral incongruence assists in explaining the relationship between religiosity and PPU, but future research should consider other manifestations of CSB beyond PPU. Attention should also be given to examining other religiosity and spirituality constructs and obtaining more diverse samples in research on CSB, religiosity, and spirituality.
\end{abstract}

\section{KEYWORDS}

compulsive sexual behavior, religiosity, spirituality, systematic review, hypersexuality, sex addiction

Diagnostic categories that capture symptoms of compulsive, addictive, impulsive, or out-ofcontrol sexual behavior have been omitted from nosologies of mental disorders for several decades. Recently, however, the International Classification of Diseases (11th ed.; ICD-11) included Compulsive Sexual Behavior Disorder (CSBD) as an impulse control disorder (Kraus et al., 2018; World Health Organization [WHO], 2018). Individuals suffering from CSBD experience significant impairment or distress due to failure to control sexual thoughts and impulses resulting in repetitive sexual behavior (WHO, 2018). CSBD has several behavioral manifestations, including paid sexual services, pornography use, masturbation, and partnered sexual activities (Antons \& Brand, 2021).

The creation of the CSBD diagnosis reflects the substantial progress in our scientific understanding of out-of-control sexual behavior (Grubbs, Hoagland, et al., 2020), but the diagnosis has also been the subject of heated debate (Kraus et al., 2018). Indeed, proposals to include Hypersexual Disorder, a syndrome similar to CSBD, in the Diagnostic and Statistical Manual of Mental Disorders (5th ed.; DSM-5) were not successful because there was deemed 
to be insufficient scientific evidence to support the creation of a new diagnosis (Kafka, 2014). Even among researchers that support creating a diagnosis, there is debate on whether CSBD should be conceptualized as an impulsive, compulsive, or addictive disorder (Coleman et al., 2018; Kraus et al., 2016).

There is also debate around the risk of CSBD misdiagnosis, especially the possibility that sexual behavior in conflict with one's moral values may be misdiagnosed as CSBD. For instance, a person engaging in sexual behavior deemed immoral by a religious or cultural community (such as masturbation, same-sex sexual behavior, or extra-marital sex) may be diagnosed with a "disorder" when their distress and impairment is more accurately the result of social stigma. To address this concern, the ICD-11 diagnosis specifies as a rule-out that distress must not be solely due to moral judgments (WHO, 2018). However, debate remains around whether this exclusion criterion is sufficient to prevent inappropriate diagnosis. Research is needed to understand how religious and cultural factors should be addressed, ruled-out, or integrated into the diagnosis and treatment of CSBD.

\section{THE PORNOGRAPHY PROBLEMS DUE TO MORAL INCONGRUENCE MODEL}

The Pornography Problems due to Moral Incongruence model (PPMI; Grubbs, Perry, Wilt, \& Reid, 2019) was developed to explain the contribution of both moral conflict and behavioral dysregulation to the development of problematic pornography use (PPU). In the present paper, PPU is conceptualized as a manifestation of CSB involving use of pornography that leads to distress and impairment (Antons \& Brand, 2021). The PPMI asserts that self-perceived PPU may arise from moral incongruence (MI), or a conflict between a person's moral or religious disapproval of pornography and their pornography use behavior. This represents a specific pathway for the development of self-perceived PPU, which needs to be distinguished (or ruled out) from the "dysregulated use" pathway represented in the CSBD criteria (Grubbs, Perry, et al., 2019).

Clinicians working with clients self-reporting PPU may encounter multiple clinical presentations: clients exhibiting distress entirely due to $\mathrm{MI}$, clients that only experience dysregulated use (and likely meet CSBD criteria), and clients that experience both MI and dysregulated use (Kraus \& Sweeney, 2019). Because sexually restrictive religious beliefs are a strong correlate of moral disapproval of pornography use, and therefore of MI, understanding the relationship between religiosity and CSB is important both conceptually and diagnostically.

While the PPMI model provides a useful framework for understanding the role of religious and moral values in diagnosing CSBD, the model has not been expanded to include other manifestations of CSB, such as compulsive partnered sex or sexual thoughts. PPU is likely the most common manifestation of CSBD, with some research suggesting it represents $81 \%$ of diagnosable cases (Reid et al., 2012), but it nevertheless does not represent all cases. It especially does not represent cases where risk of sexually transmitted infections (STIs) or illegal behavior are considerations. It is therefore important to understand how religious and moral values relate to all manifestations of CSB.

\section{EXISTING REVIEWS OF THE LITERATURE}

Current reviews of the literature examining the relationship between religiosity and CSB have found few studies on the topic. A review of the literature published by Karaga et al. (2016) found only 14 empirical studies. In addition, the authors noted that multiple articles did not examine CSB, but other constructs that are often wrongly conflated with $\mathrm{CSB}$, such as frequency of pornography use or reasons for using pornography. The review did identify a small number of studies reporting a positive correlation between religiosity and self-perceived PPU, but no relationship between religiosity and pornography use. This result suggests that religious individuals may be more likely to perceive their sexual behavior as problematic or addictive.

More recently, a review from Grubbs, Perry, et al. (2019) provided a meta-analysis examining the direct association between PPU and religiosity, as well as the mediated relationship between these constructs via moral incongruence. Results of the meta-analysis indicated that while religiosity predicted self-perceived pornography related problems (aggregate $r=0.31$ ), the mediated relationship explained a greater portion of the variance with religiosity predicting moral incongruence (aggregate $r=0.65$ ), which in turn predicted self-perceived pornography related problems (aggregate $r=0.67$ ). While this study provides invaluable data on the role of religiosity in PPU, the authors' focus on PPU leaves out other manifestations of CSB. Additionally, most of the studies included in Grubbs, Perry, et al. (2019) assessed religiosity with measures of religious participation and belief salience. A review considering various religiosity constructs would aid in understanding what other forms of religiosity are connected to CSB. Lastly, both Karaga et al. (2016) and Grubbs, Perry, et al. (2019) did not systematically examine the demographics and methodology of the studies they reviewed. It is therefore unclear what gaps in the current literature remain in terms of representativeness and methodological rigor.

\section{CSB AND SPIRITUALITY}

While there is a link between CSB and religiosity, it is also important to consider spirituality. Religiosity and spirituality are connected, but distinct constructs (Pargament, 1997; Zinnbauer et al., 1999) that may have unique relationships with CSB. Religiosity is conceptualized as one's relationship with the sacred and is often operationalized as the beliefs and practices of a particular religious perspective or group 
(Iannello et al., 2019). Conversely, spirituality may be experienced both within and outside of a particular religious context (Benson, Roehlkepartain, \& Rude, 2003) and is characterized by one's reason for existing, a desire for transcendence, and a sense of interconnectedness (King \& Boyatiz, 2015). Although many may identify as religious and spiritual, around $27 \%$ of individuals identify as only spiritual (Lipka \& Gecewicz, 2017), suggesting that these constructs are different. To the extent that aspects of spirituality are distinct from religiosity, there may be novel relationships between these constructs and CSB. Additionally, CSB has been linked to constructs that have ties to spirituality, such as lack of meaning/purpose (Hall, 2011) and lack of mindfulness (Collins \& Adleman, 2011). These considerations suggest a connection between CSB and spirituality; however, a systematic review is needed to clarify what aspects of spirituality are more associated with CSB.

\section{THE PRESENT STUDY}

This review of the literature aims to systematically examine all studies that have investigated the association between religiosity, spirituality, and CSB and provide recommendations for future areas of research. The present review considers all behavioral manifestations and conceptualizations of CSB and CSBD, including PPU, and uses CSB as an umbrella term for the many conceptualizations of the disorder (i.e., out-of-control sexual behavior, hypersexuality, sexual addiction). In the years since the Karaga et al. (2016) review, research productivity on this topic has proliferated and is seeing increased relevance with the recent inclusion of CSBD in the ICD-11. While Grubbs, Perry, et al. (2019) provided a valuable review of the relationship between religiosity and PPU, there are several notable research areas that have yet to gain sufficient attention regarding the CSB religiosity link. This includes demographic summaries of the literate, reviews of studies that examine other forms of CSB beyond PPU, and an evaluation of the methodological rigor of studies in the field. This review will fill in the gaps of previous reviews and provide an update on the rapidly changing research examining the relationship between CSB, religiosity, and spirituality.

\section{METHODS}

A systematic review of CSB, religion, and spirituality research published through August 1, 2021, was conducted using the Preferred Reporting Items for Systematic Reviews and Meta-analyses (PRISMA) guidelines (Moher et al., 2009). A quantitative meta-analysis of results was not conducted as the vast diversity of measures and methods used in research on CSB, religiosity, and spirituality render such an analysis impossible to conduct in a meaningful manner. Although Grubbs, Perry, et al. (2019) was able to conduct a meta-analysis focused on PPU and MI, there is a greater continuity among measures of these constructs in the literature compared to studies examining the association between other CSB and religiosity constructs. Despite these limitations, a detailed qualitative synthesis remained feasible and meaningful. The search was performed in two databases (APA PsycINFO and PubMed), supplemented by Google scholar searches, cross-referencing articles, and consultation with researchers in the field. The first three authors independently coded each article. Each article was then rereviewed by the first two authors to check for coding errors. A group discussion among the first three authors settled any disagreements on the inclusion or coding of studies. A full summary of the review process can be seen in Fig. 1.

\section{Search terms}

Search terms included the following items: "hypersexuality and relig," "hypersexuality and spirit"," "compulsive sexual behavior and relig*," "compulsive sexual behavior and spirit"," "sexual addiction and relig"," "sexual addiction and spirit*," "sexual impulsivity and relig*," "sexual impulsivity and spirit", "sexual compulsivity and relig*," and "sexual compulsivity and spirit*" Entering these terms into PsycInfo and PubMed produced 1,099 results. The present authors analyzed the titles and abstracts of the identified documents and included those that were relevant to both CSB and religiosity or spirituality. This process identified 178 articles. Supplemental literature searches, cross-references, and consultations with researchers in the field revealed another 38 articles for review, producing a total of 216 manuscripts.

\section{Exclusion and inclusion criteria}

First, 73 duplicate articles were removed from the analysis, reducing the number of eligible papers to 143 . Twenty-eight additional papers were not published in academic journals and were removed. The remaining articles were subject to title and abstract analysis. To be included, studies needed to involve a quantitative empirical analysis of CSB and religiosity or spirituality (e.g., literature reviews, commentaries, and qualitative studies were removed). Studies met criteria if they included at least one measure of religiosity or spirituality and CSB, measured CSB in a sample of religious or spiritual individuals, or measured religiosity or spirituality in a sample of individuals with self-reported CSB (e.g., selfidentified "pornography addicts"). If non-religious participants were included in a study, the study remained eligible for review so long as the paper measured a religiosity or spirituality construct. Studies examining sexual behavior (e.g., pornography use or hiring sex workers), and not CSB, were removed from analysis, as well as studies examining attitudes toward others with CSB.

Using the above criteria, 62 articles were removed from analysis using title and abstract screening. If there was not enough information in the title and abstract to determine whether a study should be excluded from the main analysis, the article remained eligible for full-text review. This process left 81 articles eligible for full-text review. Of these 81 papers, 35 did not meet inclusion criteria and were removed from analysis. Thus, the present search produced 46 articles, 


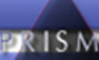

PRISMA 2009 Flow Diagram
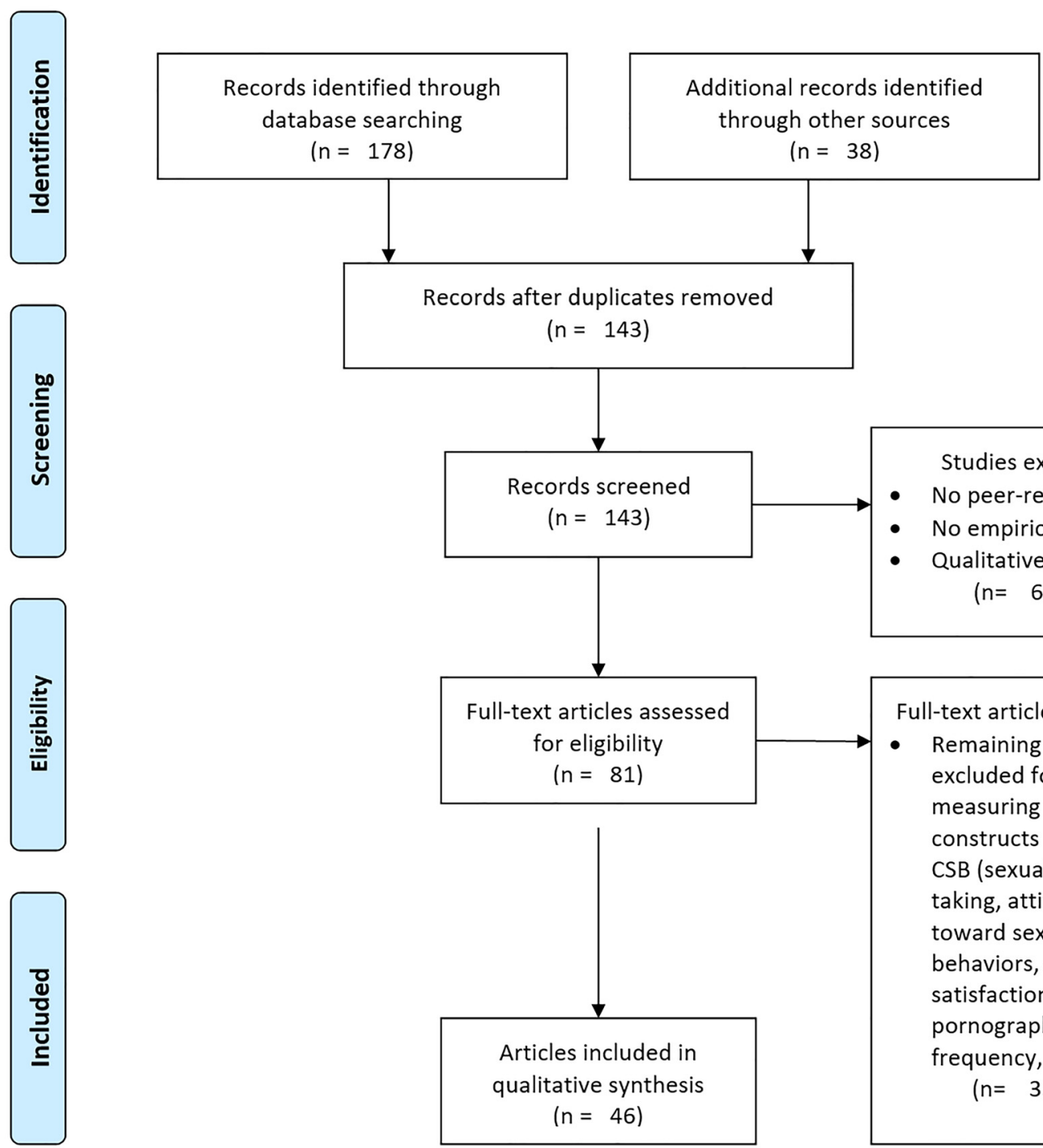

Studies excluded

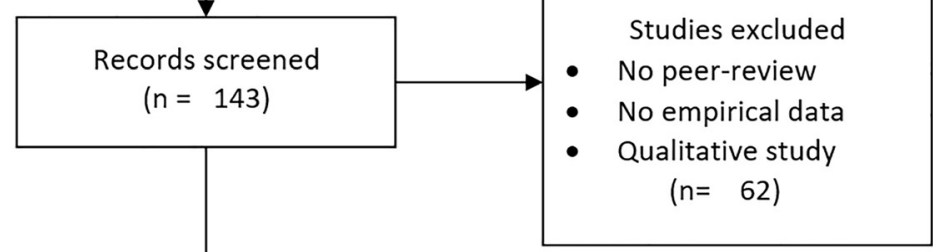

Full-text articles assessed for eligibility $(n=81)$

Full-text articles excluded

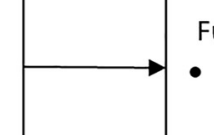

Remaining articles excluded for measuring sexuality

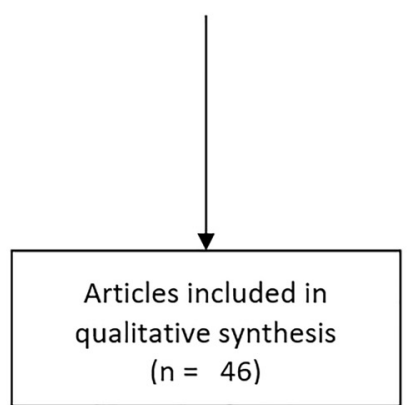
constructs besides CSB (sexual risktaking, attitudes toward sexual behaviors, sexual satisfaction, pornography use frequency, etc.) $(n=35)$

Fig. 1. CSB, religiosity, and spirituality article selection process

Note. Preferred Reporting Items for Systematic Reviews and Meta-analyses (PRISMA) flow diagram illustrating article selection process.

subsuming 59 separate studies, analyzing compulsive sexual behavior (CSB) and religiosity or spirituality. A diagram of this process can be seen in Fig. 1.

\section{RESULTS}

Publication dates of the studies included in this review are shown in Fig. 2. Before 2005, there was no empirical research on CSB, religiosity, and spirituality that met the present review's inclusion criteria. From 2005 to 2009, there were two $(3 \%)$ studies published that met review criteria. From 2010 to 2014, 6 (10\%) studies were published, averaging 1.2 publications per year. From 2015 to 2019, 37 (63\%) studies were published, averaging 7.4 publications per year. Finally, from 2020 to August 1st, 2021, 14 (24\%) studies were published.

\section{Demographic summary}

A diverse range of ages were represented in the literature. Of the 59 studies in the review, 38 (65\%) examined participants of various ages, $12(20 \%)$ limited their sample to college students, and nine (15\%) used an adolescent sample. Regarding gender, 43 (73\%) studies included both men and women. Fourteen (24\%) examined men exclusively and two (3\%) examined women exclusively. A few studies were heavily skewed toward men or women: two had a sample 


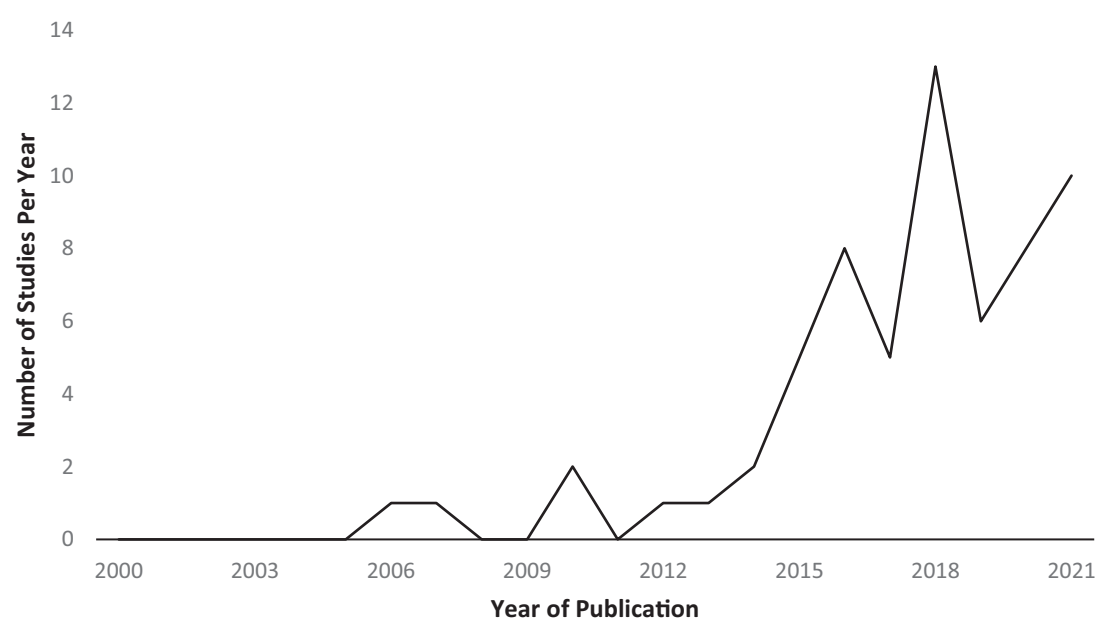

Fig. 2. CSB, religiosity, and spirituality studies per year

Note. Publication dates of included studies through August 1, 2021. Data point for 2021 estimated by multiplying the rate of publications each month for 2021 by 5 and adding the product to the number of publications (6) from January 1, 2021 to August 1, 2021.

consisting of $75 \%$ or more men $(3 \%)$, and another was composed of $75 \%$ or more women $(1 \%)$. One (1\%) study reported data on the prevalence of transgender participants. Twenty-nine (49\%) studies reported demographic information on sexual orientation. Of these, two (7\%) used exclusively heterosexual samples and three (10\%) limited their sample to men who have sex with men.

Forty $(68 \%)$ of the 59 studies in the present review reported demographic information on race and ethnicity. Of these 40 studies, 37 (93\%) had samples with White participants as the largest race/ethnic group and two (5\%) had samples where other race/ethnicity categories were the largest group. The remaining study (2\%) reporting information on race and ethnicity did not provide enough detail to determine which group represented the largest portion of the sample. Among the 37 studies with majority White samples, White participants comprised more than $75 \%$ of the sample in nineteen (51\%) studies and $50-75 \%$ of the sample in seventeen (46\%) studies. One study (3\%) had Whites as the largest sampled race, but these participants did not exceed $50 \%$ of the sample.

Fifty-five (93\%) of the 59 studies reported the country in which they were conducted. Studies took place in several countries, though a majority were conducted in the United States $(n=34,61 \%)$, while seven were conducted in Israel (13\%), five in Croatia (9\%), and four in Poland (7\%). One study was multinational (2\%), and the remaining four were in each of the following countries: New Zealand, Sweden, Spain, and the United Kingdom.

\section{Study type}

Study types identified in the present review were classified as either cross-sectional samples or longitudinal studies. Fortyseven $(80 \%)$ studies in the present review used a crosssectional sample. Twelve (20\%) studies were longitudinal, comprising various demographic groups and study lengths, including an adolescent sample, a sample of Jewish men, and a sample of undergraduates. The lengths of longitudinal studies ranged from six months to three years, with two to six data collection waves. One study in the current review was experimental. Four studies (4\%) using a cross-sectional sample also had clinical or treatment-seeking samples.

\section{Prevalence of PPU among religious and spiritual individuals}

There has been little research examining the prevalence of CSB among religious and/or spiritual individuals. However, there have been four studies that examined the connection between religiosity and PPU in non-probability samples matched to national norms (U.S. or Poland) (Grubbs, Grant, et al., 2018; Grubbs, Kraus, et al., 2019; Grubbs, Lee, et al., 2020; Lewczuk et al., 2020). Three of these studies were conducted in the United States and one was conducted in Poland. An additional study conducted in Poland assessed the same construct and used a nationally representative sample (Lewczuk et al., 2021). All five studies reported that men were more likely to agree with the statement "I [believe I] am addicted to [internet] pornography" or "I believe I am addicted to internet pornography" than women. Each of the five articles also reported that religiousness was predictive of endorsing the above statement. Specific prevalence rates for agreeing with the statement "I [believe I] am addicted to [internet] pornography" ranged from $3 \%$ to $5 \%$ for women and $9 \%-18 \%$ for men (Grubbs, Grant, et al., 2018; Grubbs, Kraus, et al., 2019; Grubbs, Lee, et al., 2020; Lewczuk et al., 2020; Lewczuk et al., 2021).

\section{Measurement of CSB}

The present review identified a total of 25 CSB measures that were used across the studies analyzed, including 15 validated instruments, 8 modified or adapted instruments, and 2 instruments categorized as other (e.g., one- or two-item measures). Three validated CSB instruments were used in 
five or more studies: the Cyber-Pornography Use Inventory9 (CPUI-9; Grubbs, Volk, et al., 2015) was used in 15 studies, the Hypersexual Behavior Inventory-19 (HBI-19; Reid et al., 2011) was used in seven studies, and the Individual-Based Compulsive Behavior Scale (I-CSB; Efrati \& Mikulancer, 2018) was used in seven studies. The remaining 22 measurement tools or instruments were used at most four times $(n=3)$, but more commonly measures were used once $(n=13)$ or twice $(n=3)$. Most studies measured CSB with one instrument $(n=50)$, but some used two $(n=6)$, three $(n=2)$, or four $(n=2)$ different instruments. The most common CSB manifestation measured in the studies reviewed was PPU, with 32 (54\%) studies examining this construct.

\section{Measures of religiosity}

Over half of the studies in the present review used nonvalidated one-, two-, or three item measures of religiosity $(n=30,51 \%)$. The remaining studies used validated or adapted measures of religiosity. There were 12 validated and four adapted measures of religiosity. The most commonly used religiosity instrument was an aggregate of two adapted religiosity measures: the Religious Belief Salience Scale (RBSS; Blaine \& Crocker, 1995) and the Adapted Religious Participation Measure (ARPM; Exline, Yali, \& Sanderson, 2000). This measure was used 11 times and the adapted RBSS alone was used in an additional study (Wilt, 2016). Nine studies used a single religious affiliation question (e.g., "what is your religion?") and six studies used a three-item measure that examined multiple aspects of religiosity (e.g., religious service attendance) that can be found in Grubbs, Grant, et al. (2018). The remaining validated and adapted measures were used two $(n=4)$, three $(n=1)$, or four times $(n=1)$, but more commonly measures were used once $(n=10)$. Most studies measured religiosity with one measure or instrument $(n=43,73 \%)$.

Across these measures of religiosity, 14 constructs were identified. The most commonly assessed constructs were religious participation (e.g., "How often do you attend religious services?") assessed in 23 (40\%) studies, general religiosity (e.g., "I consider myself religious.") assessed in 19 (32\%) studies, and belief salience (e.g., "Being a religious person is important to me.") assessed in 13 (25\%) studies. The remaining 11 constructs were assessed in one to four studies, some of which were derived from validated measures and others that were not. Notably, many studies and instruments measured more than one construct. Other assessed constructs included religious/spiritual struggles $(n=5)$, religious coping $(n=3)$, religious commitment/community ties $(n=3)$, faith in God $(n=$ $4)$, extrinsic/intrinsic religiosity $(n=3)$, and scrupulosity $(n=2)$. It is notable that several studies described measuring general aspects of religiosity. However, general religiosity is not clearly defined in the literature. Therefore, a meaningful quantitative summary for this construct is not obtainable.

\section{Measures of spirituality}

Seven studies (11\%) measured spirituality constructs. Three different validated measures were used across six studies and a seventh study used a non-validated single item measure of spirituality. The most common measure was the Religious and Spiritual Struggles Scale (RSS; Exline, Pargament, Grubbs, \& Yali, 2014), which was used in four of the six studies (66\%). The remaining two measures were each used once, those being the Spiritual Well-Being Scale (Ellison, 1983) and the Spiritual Assessment Scale (Howden, 1992). One additional study used a single-item asking, "How spiritual are you?” (Borgogna, Isacco, \& McDermott, 2020).

\section{The relationship between CSB, religiosity, and spirituality}

Of the 59 studies in the present review, 46 (78\%) found a significant relationship between CSB and religiosity or spirituality, while 13 studies (22\%) found no significant relationship. Of the 46 studies reporting a relationship, 35 exclusively reported positive associations (76\%). Four studies reported both positive and negative relationships between CSB and different religiosity constructs (9\%), generally finding that high CSB was negatively related to positive religious constructs such as healthy religious coping (Giordano \& Cecil, 2014). Six studies (13\%) reported only significant differences between religious and non-religious groups on measures of CSB and one (2\%) study reported a significant relationship but did not specify the direction of this association (Ross et al., 2012). Nearly all direct associations between CSB and religiosity had a small to moderate effect size, while a few had a large effect size (see Table 1).

Because a wide variety of constructs and statistical analyses were used in these studies, it was not possible to conduct a meta-analysis of the data. In lieu of this consideration, one way to provide greater clarity to this heterogeneous literature is to examine religiosity and CSB constructs that have been compared multiple times across the literate. To this end, studies that included the most commonly used CSB contracts were examined: the CPUI-9 (Grubbs, Volk, et al., 2015), the HBI-19 (Reid et al., 2011), and the I-CSB (Efrati \& Mikulancer, 2018). Findings of this examination are described below and in Table 1.

\section{Relationship between the CPUI-9 and religiosity}

The CPUI-9 is a 9-item scale that assesses perceived addiction to pornography via three subscales: access efforts, perceived compulsivity, and emotional distress (Grubbs, Volk, et al., 2015). Fourteen studies utilized the CPUI-9, and all found positive associations between this measure and measures of religiosity. Religiosity constructs that were positively associated with the CPUI-9 included an aggregate measure of belief salience and religious participation $(n=7)$, religious/spiritual struggles $(n=2)$, a standalone belief salience measure $(n=1)$, certainty in God's existence $(n=1)$, religious scrupulosity $(n=1)$, and the composite scale score of the Dimensions of Religiosity Scale 


\begin{tabular}{|c|c|c|c|c|}
\hline Authors/Year & $\begin{array}{l}\text { Sample/Country } \\
\text { Age }(\text { Mean/SD) }\end{array}$ & $\begin{array}{c}\text { Gender/Race/ } \\
\text { Sexual Orientation }\end{array}$ & $\begin{array}{l}\text { CSB \& Religiosity/ } \\
\text { Spirituality Measure }\end{array}$ & Results \\
\hline $\begin{array}{l}\text { Abell, Steenbergh, and Boivin } \\
\text { (2006) }\end{array}$ & $\begin{array}{c}N=125 ; \text { undergraduates } \\
\text { US } \\
M=20.67, \mathrm{SD}=4.51\end{array}$ & $\begin{array}{l}100 \% \text { men } \\
\text { Unreported } \\
\text { Unreported }\end{array}$ & $\begin{array}{c}\text { SCS Adapted, G-SAST, SWBS, \& } \\
\text { SBI-15R }\end{array}$ & $\begin{array}{l}\text { Small positive correlation between } \\
\text { the SCS adapted and the SBI-15R } \\
(r=0.22, P<0.05) \text { and two small } \\
\text { negative correlations between the } \\
\text { G-SAST and SWBS }(r=-0.21, P< \\
0.05) \text {, as well as the SBI-15R }(r= \\
\quad-0.17, P<0.05) .\end{array}$ \\
\hline Levert (2007) & $\begin{array}{c}N=120 \\
\mathrm{US} \\
M=39.51, \mathrm{SD}=\text { unreported }\end{array}$ & $\begin{array}{c}100 \% \text { men } \\
77.5 \% \text { Caucasian } \\
88 \% \text { heterosexual, } 3.3 \% \\
\text { homosexual, } 8.3 \% \text { bisexual }\end{array}$ & SCS \& religious affiliation & $\begin{array}{l}\text { Christian men }(30.6 \%) \text { were more } \\
\text { likely to report feeling that their } \\
\text { pornography use is compulsive as } \\
\text { compared to non-Christian men } \\
(14.7 \% ; \chi 2=15.01, P<0.001)\end{array}$ \\
\hline Coleman et al. (2010) & $\begin{array}{c}N=2,716 \\
\mathrm{US} \\
M=29, \mathrm{SD}=8.3\end{array}$ & $\begin{array}{c}100 \% \text { men } \\
26.8 \% \text { White } \\
100 \% \text { men who have sex with men } \\
(\text { MSM })\end{array}$ & $\begin{array}{l}\text { CSBI \& single item specific to study } \\
\text { regarding religiosity }\end{array}$ & $\begin{array}{l}\text { MSM who reported being very } \\
\text { religious had significantly higher } \\
\text { CSB scores than MSM that did not } \\
\text { consider themselves religious } \\
(F(2,2,693)=7.01, P<0.001) .\end{array}$ \\
\hline Skegg et al. (2010) & $\begin{array}{c}N=940 \\
\mathrm{NZ} \\
M=32, \mathrm{SD}=0\end{array}$ & $\begin{array}{c}50.6 \% \text { men and } 49.6 \% \text { women } \\
\text { Unreported } \\
\text { Unreported }\end{array}$ & $\begin{array}{l}\text { CSB \& single items specific to study } \\
\text { regarding religiosity }\end{array}$ & $\begin{array}{l}\text { Men reporting out of control sexual } \\
\text { experiences (OSCE; 11.7\%), rated } \\
\text { religion as "very important," which } \\
\text { was similar to religiosity ratings } \\
\text { from other men that did not report } \\
\text { OSCE. Similarly, there was not a } \\
\text { significant difference among } \\
\text { religious women that did, or did } \\
\text { not, report OSCE. }\end{array}$ \\
\hline Ross et al. (2012) & $\begin{array}{c}N=1,913 \\
\text { Sweden } \\
\text { Men }(M=28.91, \mathrm{SD}=12.67) \\
\text { Women }(M=28.12, \mathrm{SD}=9.91)\end{array}$ & $\begin{array}{l}34.3 \% \text { men and } 65.7 \% \text { women } \\
\text { Unreported } \\
\text { 87\% heterosexual men; } 80 \% \\
\text { heterosexual women }\end{array}$ & $\begin{array}{l}\text { CSB items specific to study, \& } \\
\text { religious affiliation }\end{array}$ & $\begin{array}{l}\text { Internet sexual problems were } \\
\text { significantly predicted by degree of } \\
\text { religiosity as part of a larger logistic } \\
\text { regression. An effect size and the } \\
\text { direction of the relationship were } \\
\text { not reported. }\end{array}$ \\
\hline Thompson and Morrison (2013) & $\begin{array}{c}N=571 ; \text { undergraduates } \\
\text { US } \\
\text { Unreported }\end{array}$ & $\begin{array}{l}100 \% \text { men } \\
\text { Unreported } \\
\text { Unreported }\end{array}$ & $\begin{array}{l}\text { SCS \& single item specific to study } \\
\text { regarding religiosity }\end{array}$ & $\begin{array}{l}\text { Participation in a religious group } \\
\text { during the last academic year was } \\
\text { not significantly correlated with } \\
\text { sexual compulsivity. } \\
\text { (continued) }\end{array}$ \\
\hline
\end{tabular}


Table 1. Continued

\begin{tabular}{|c|c|c|c|c|}
\hline Authors/Year & $\begin{array}{l}\text { Sample/Country } \\
\text { Age }(\text { Mean/SD) }\end{array}$ & $\begin{array}{c}\text { Gender/Race/ } \\
\text { Sexual Orientation }\end{array}$ & $\begin{array}{l}\text { CSB \& Religiosity/ } \\
\text { Spirituality Measure }\end{array}$ & Results \\
\hline Dhuffar and Griffiths (2014) & $\begin{array}{c}\qquad N=102 \\
\text { UK } \\
\text { Grouped into young adults (47.1\% } \\
\text { between ages } 18-29) \text { and older } \\
\text { adults } 53.9 \% \text { ages } 30+)\end{array}$ & $\begin{array}{c}100 \% \text { women } \\
87.3 \% \text { Caucasian } \\
87.3 \% \text { heterosexual }\end{array}$ & $\begin{array}{l}\text { HBI-19, HBCS, HDQ, Adapted } \\
\text { Internet Related Activities, religious } \\
\text { affiliation, \& religious belief }\end{array}$ & $\begin{array}{l}\text { Religious beliefs (belief vs. no belief) } \\
\text { and affiliation had no influence on } \\
\text { consequences of sexual behaviors as } \\
\text { predictors of shame. There were no } \\
\text { significant correlations between } \\
\text { religious beliefs and measures of } \\
\text { CSB in the study. }\end{array}$ \\
\hline Giordano and Cecil (2014) & $\begin{array}{c}N=235 ; \text { undergraduates } \\
\text { US } \\
M=20.91, \mathrm{SD}=3.56\end{array}$ & $\begin{array}{l}58 \% \text { men and } 42 \% \text { women } \\
55.3 \% \text { White/Caucasian } \\
94.5 \% \text { heterosexual }\end{array}$ & $\begin{array}{l}\text { HBI-19, religious affiliation, \& Brief } \\
\text { RCOPE }\end{array}$ & $\begin{array}{l}\text { Purpose/Meaning subscale of the } \\
\text { SAS }(B=-1.09, \beta=-0.21, t= \\
-2.60, P=0.010) \text {, and the negative } \\
\text { religious coping subscale of } \\
\text { BRCOPE }(B=0.53, \beta=0.17, t= \\
2.55, P<0.05) \text { significantly } \\
\text { contributed to the explained } \\
\text { variance in hypersexuality. }\end{array}$ \\
\hline $\begin{array}{l}\text { Carvalho, Štulhofer, Vieira, and } \\
\text { Jurin (2015) }\end{array}$ & $\begin{array}{c}N=4,597 \\
\text { Croatia } \\
M=31.1, \mathrm{SD}=9.67\end{array}$ & $\begin{array}{c}43.5 \% \text { men } \\
\text { Unreported } \\
66.7 \% \text { heterosexual }\end{array}$ & $\begin{array}{l}\text { HBCS, single items regarding CSB } \\
\text { and religiosity specific to study }\end{array}$ & $\begin{array}{l}\text { Among women, a lower religiosity } \\
(\mathrm{AOR}=0.81, P<0.05) \text { decreased } \\
\text { the odds of belonging to the sexual } \\
\text { desire/activity cluster }(M=0.11 \text {, } \\
\mathrm{SD}=1.59) \text {. The same relationship } \\
\text { did not hold for the men in the } \\
\text { sample }(M=0.96, \mathrm{SD}=1.61)\end{array}$ \\
\hline $\begin{array}{l}\text { Grubbs, Exline, et al. } \\
\quad(2015 \text {, Study 1) }\end{array}$ & $\begin{array}{c}N=331 ; \text { undergraduates } \\
\text { US } \\
\qquad M=19.5, \mathrm{SD}=1.9\end{array}$ & $\begin{array}{c}68.9 \% \text { men and } 31.1 \% \text { women } \\
67 \% \text { White/Caucasian } \\
88 \% \text { heterosexual }\end{array}$ & $\begin{array}{l}\text { CPUI-9, religious affiliation, \& } \\
\text { aggregate score of RBSM \& ARPM }\end{array}$ & $\begin{array}{l}\text { Positive associations between } \\
\text { religiosity and perceived problems } \\
\text { related to pornography and CPUI }= \\
9(r=0.25, P<0.01 \text { for both }) .\end{array}$ \\
\hline $\begin{array}{l}\text { Grubbs, Exline, et al. } \\
\quad(2015 \text {, Study 2) }\end{array}$ & $\begin{array}{c}N=97 ; \text { undergraduates } \\
\text { US } \\
M=19.5, \mathrm{SD}=1.3\end{array}$ & $\begin{array}{c}50.5 \% \text { men and } 49.5 \% \text { women } \\
54 \% \text { White/Caucasian } \\
89 \% \text { heterosexual }\end{array}$ & $\begin{array}{l}\text { CPUI-9, religious affiliation, \& } \\
\text { aggregate score of RBSM \& ARPM }\end{array}$ & $\begin{array}{l}\text { Positive association between } \\
\text { religiosity and CPUI- } 9(r=0.35 \text {, } \\
\qquad P<0.01)\end{array}$ \\
\hline $\begin{array}{l}\text { Grubbs, Exline, et al. } \\
\quad(2015 \text {, Study } 3)\end{array}$ & $\begin{array}{c}N=208 \\
\mathrm{US} \\
M=31.8, \mathrm{SD}=10.6\end{array}$ & $\begin{array}{c}65.4 \% \text { men and } 34.6 \% \text { women } \\
79 \% \text { White/Caucasian } \\
79 \% \text { heterosexual }\end{array}$ & $\begin{array}{l}\text { CPUI-9, SCS, religious affiliation, \& } \\
\text { aggregate score of RBSM \& ARPM }\end{array}$ & $\begin{array}{l}\text { Positive associations between } \\
\text { religiosity and perceived } \\
\text { compulsivity related to } \\
\text { pornography }(r=0.38, P<0.01) \\
\text { and between religiosity and CPUI-9 } \\
\qquad(r=0.48, P<0.01) \\
\text { (continued) }\end{array}$ \\
\hline
\end{tabular}




\begin{tabular}{lccc}
\hline \multirow{2}{*}{ Authors/Year } & Sample/Country & Gender/Race/ & CSB \& Religiosity/ \\
& Age (Mean/SD) & Sexual Orientation & Spirituality Measure \\
\hline Hook et al. (2015, Study 2) & $N=191$; undergraduates & $34.4 \%$ men and $65.6 \%$ women & HBI-19, religious affiliation, \& \\
& $M=24.4, \mathrm{SD}=5.3$ & $41.1 \%$ Black & Spiritual Struggle Scale
\end{tabular}

Bradley Grubbs, Uzdavines, Exline, and Pargament (2016)

$$
N=713
$$

$M=30.2, \mathrm{SD}=9.9$

Gola, Lewczuk, and Skorko (2016)

$$
\begin{gathered}
N=569 \\
\text { Poland } \\
M=28.71, \mathrm{SD}=6.36
\end{gathered}
$$

$N=295$; undergraduates

US

$M=20.25, \mathrm{SD}=3.95$

$$
N=157
$$

US

HHR $(M=39.5, \mathrm{SD}=13.5$ $\operatorname{HNR}(M=44.3, \mathrm{SD}=10.5)$

Reid et al. (2016)
Griffin et al. (2016)

$51.9 \%$ men, $47.4 \%$ women, $0.7 \%$ other/prefer not to say 78\% White/Caucasian Unreported

$100 \%$ men

$100 \%$ White/Caucasian

$100 \%$ heterosexual

$77.9 \%$ women; remaining data not reported

$51 \%$ White/Caucasian

$84.7 \%$ heterosexual
$100 \%$ men

HHR: 94\% White/Caucasian

94\% heterosexual

HNR: 86\% White/Caucasian $81 \%$ heterosexual
CPUI-9, religious affiliation, \& single item specific to study regarding religiosity

SAST-R (Polish adaptation) \& religiosity items specific to study

HBI-19 \& RSS

\section{HBI-19, HBCS, clinical interview, \&} RCI

HBI-19 correlates with the spiritual struggles scale $(r=0.37, P<0.01)$.

Sexual congruence was negatively to the SSS $(r=-0.32, P<0.01)$, and the SSS was also negatively related to selfforgiveness $(r=-0.19, P<0.05)$.

CPUI-9 full scale and certainty of belief $(r=0.24, P<0.01)$, CPUI- 9 Emotional Distress and certainty of belief $(r=0.29, P<0.01)$, and CPUI-9 Perceived Compulsion and certainty of belief $(r=0.14, P<$ 0.01 ). Belief in God is predictive of perceived addiction to Internet pornography.

Found positive associations $(N=$ 476; $r=0.40, P<0.001)$ between religiousness and perceived problems associated with pornography use.

Main effects of hypersexual behavior on demonic $(\beta=0.68$, $P=0.043)$, ultimate meaning $(\beta=$ $0.66, P=0.002)$, moral $(\beta=0.71$ $P=0.002)$, doubt $(\beta=0.88, P<$ $0.001)$, and interpersonal struggle ( $\beta=0.80, P=0.002)$, but not divine struggle $(\beta=0.26, P=$ 0.222). For moral, doubt, and interpersonal struggles, the associations between hypersexual behavior and spiritual struggle appeared strongest for those who perceived their sexual values and behavior as incongruent.

No significant relationship between scores on the RCI and the HBI-19 or HBCS3 in a sample of religious assessed for hypersexual disorder as part of the DSM-5 Field Trial. 


\section{Table 1. Continued}

\begin{tabular}{|c|c|c|c|c|}
\hline Authors/Year & $\begin{array}{l}\text { Sample/Country } \\
\text { Age (Mean/SD) }\end{array}$ & $\begin{array}{c}\text { Gender/Race/ } \\
\text { Sexual Orientation }\end{array}$ & $\begin{array}{l}\text { CSB \& Religiosity/ } \\
\text { Spirituality Measure }\end{array}$ & Results \\
\hline Štulhofer et al. (2016) & $\begin{array}{c}N=1998 \\
\text { Croatia } \\
M=34.7, \mathrm{SD}=9.83\end{array}$ & $\begin{array}{c}100 \% \text { men } \\
100 \% \text { Croatian } \\
66.4 \% \text { exclusively heterosexual }\end{array}$ & $\begin{array}{l}\text { HBCS, HDSI, TSO, Faith in God } \\
\text { measure, \& single items specific to } \\
\text { study regarding religiosity }\end{array}$ & $\begin{array}{l}\text { Hypersexual and high sexual desire } \\
\text { groups were found to be distinct } \\
\text { and the hypersexuality group had } \\
\text { higher odds of being religious (AOR } \\
=1.32, P<0.05 \text { ). }\end{array}$ \\
\hline Timberlake et al. (2016) & $\begin{array}{c}N=802 \\
\text { Unreported } \\
M=35.62, \mathrm{SD}=13.60\end{array}$ & $\begin{array}{c}37.8 \% \text { men and } 62.2 \% \text { women } \\
\text { 68.82\% White/Caucasian } \\
\text { Unreported }\end{array}$ & $\begin{array}{l}\text { SAST, religious affiliation, single } \\
\text { item specific to study regarding } \\
\text { religiosity }\end{array}$ & $\begin{array}{l}\text { Small positive relationship between } \\
\text { service attendance and CSB }(B= \\
0.01, P<0.01) . \text { High and low CSB } \\
\text { score categories did not predict } \\
\text { religious service attendance. }\end{array}$ \\
\hline Volk et al. (2016) & $\begin{array}{c}N=358 \\
\text { Unreported } \\
M=34.13, \mathrm{SD}=10.61\end{array}$ & $\begin{array}{c}52.2 \% \text { men and } 47.8 \% \text { women } \\
\text { 83.2\% White/Caucasian } \\
\text { 87.7\% heterosexual }\end{array}$ & CPUI-9, RCI, \& RCI-Household & $\begin{array}{l}\text { Small positive relationship between } \\
\text { religiousness and problems } \\
\text { associated with pornography use. } \\
\text { Moral disapproval was found to } \\
\text { mediate the relationship between } \\
\text { religiosity and CPU-9 score; } \\
\text { however, the direct effect of } \\
\text { religiosity lost significance after } \\
\text { moral disapproval was added to the } \\
\text { model. }\end{array}$ \\
\hline Wilt et al. (2016) & $\begin{array}{c}N=1,070 ; \text { undergraduates } \\
\text { US } \\
\qquad=19.33, \mathrm{SD}=2.53\end{array}$ & $\begin{array}{c}\text { 68.4\% men and } 31.6 \% \text { women } \\
\text { 70\% White/Caucasian } \\
\text { Unreported }\end{array}$ & CPUI-9 \& Adapted RBS & $\begin{array}{l}\text { Anger toward God subscale } \\
\text { correlated with the perceived } \\
\text { compulsivity }(r=0.17, P<0.01) \\
\text { and access efforts }(r=0.30, P< \\
0.01) \text { subscales of the CPUI-9. } \\
\text { Religiosity positively correlated with } \\
\text { each subscale of the CPUI-9: } \\
\text { perceived compulsivity }(r=0.26 \text {, } \\
P<0.01) \text {, access efforts }(r=0.10, \\
P<0.01) \text {, and emotional distress } \\
(r=0.50, P<0.01) \text {. A positive } \\
\text { correlation was also present } \\
\text { between moral disapproval and } \\
\text { religiousness }(r=0.66, P<0.01) \text {. }\end{array}$ \\
\hline $\begin{array}{l}\text { Giordano, Cashwell, Lankford, } \\
\text { King, and Henson (2017) }\end{array}$ & $\begin{array}{c}N=326 ; \text { undergraduates } \\
\text { US } \\
M=23.15, \mathrm{SD}=5.03\end{array}$ & $\begin{array}{c}40.5 \% \text { men, } 58.9 \% \text { women, } 0.3 \% \\
\text { transgender, and } 0.3 \% \text { missing data } \\
51.2 \% \text { White/Caucasian } \\
91.4 \% \text { heterosexual }\end{array}$ & SAST-R \& Brief RCOPE & $\begin{array}{l}\text { Engaging in higher levels of } \\
\text { negative religious coping was } \\
\text { positively associated with scores on } \\
\text { the SAST-R. } \\
\text { (continued) }\end{array}$ \\
\hline
\end{tabular}




\begin{tabular}{|c|c|c|c|c|}
\hline Authors/Year & $\begin{array}{l}\text { Sample/Country } \\
\text { Age }(\text { Mean/SD) }\end{array}$ & $\begin{array}{c}\text { Gender/Race/ } \\
\text { Sexual Orientation }\end{array}$ & $\begin{array}{l}\text { CSB \& Religiosity/ } \\
\text { Spirituality Measure }\end{array}$ & Results \\
\hline Grubbs et al. $(2017, \text { Study } 1)^{\mathrm{a}}$ & $\begin{array}{c}\text { Time1; } N=1,519 ; \text { Time2; } N=156 ; \\
\text { undergraduates } \\
\text { US } \\
M=19.3, \mathrm{SD}=1.3\end{array}$ & $\begin{array}{c}67.2 \% \text { men, } 32.5 \% \text { women, } 0.2 \% \\
\text { other } \\
71 \% \text { White/Caucasian } \\
\text { Unreported }\end{array}$ & CPUI-9 \& RSS & $\begin{array}{l}\text { At time } 1 \text {, perceived addiction was } \\
\text { positively associated with all } 3 \\
\text { subscales of the RSS: divine } \\
\text { struggles }(r=0.29, P<0.05) \text {, moral } \\
\text { struggles }(r=0.39, P<0.05) \text {, and } \\
\text { interpersonal struggles }(r=0.23 \text {, } \\
P<0.05) \text {. These associations } \\
\text { remained at time } 2 .\end{array}$ \\
\hline Grubbs et al. $(2017 \text {, Study } 2)^{\mathrm{a}}$ & $\begin{array}{c}\text { Time1; } N=713 ; \text { Time } 2 ; N=366 \\
\text { US } \\
M=30.2, \text { SD }=9.9\end{array}$ & $\begin{array}{c}51.9 \% \text { men, } 47.4 \% \text { women }, 0.7 \% \\
\text { other/prefer not to say } \\
78 \% \text { White/Caucasian } \\
\text { Unreported }\end{array}$ & CPUI-9 \& RSS & $\begin{array}{l}\text { At time } 1 \text {, perceived addiction was } \\
\text { positively associated with all } 3 \\
\text { subscales of the RSS: divine } \\
\text { struggles }(r=0.21, P<0.05) \text {, moral } \\
\text { struggles }(r=0.37, P<0.05) \text {, and } \\
\text { interpersonal struggles }(r=0.08, \\
P<0.05) \text {. These associations } \\
\text { remained at time } 2 .\end{array}$ \\
\hline Lewczuk et al. (2017) & $\begin{array}{c}N=719 \\
\text { Poland } \\
M=26.5, \mathrm{SD}=5.93\end{array}$ & $\begin{array}{c}100 \% \text { women } \\
100 \% \text { White/Caucasian } \\
64.3 \% \text { heterosexual }\end{array}$ & $\begin{array}{l}\text { SAST-R (Polish adaptation) and } \\
\text { single items specific to study } \\
\text { regarding religiosity }\end{array}$ & $\begin{array}{c}\text { The relationship between religious } \\
\text { practices and problems associated } \\
\text { with pornography use }(r=0.25, P< \\
0.001) \text { as well as the relationship } \\
\text { between subjective religiosity and } \\
\text { problems associated with } \\
\text { pornography use }(r=0.09, P< \\
0.05) \text { were both positive and } \\
\text { significant. }\end{array}$ \\
\hline Salmerón-Sánchez et al. (2017) & $\begin{array}{c}N=124 \\
\text { Spain } \\
\text { Male sex workers }(M=23.1, \mathrm{SD}= \\
\text { 3.31) Non-male sex workers }(M= \\
23.75, \mathrm{SD}=3.79)\end{array}$ & $\begin{array}{c}100 \% \text { men } \\
100 \% \text { Hispanic } \\
100 \% \text { gay }\end{array}$ & $\begin{array}{l}\text { SCS (Spanish adaptation) \& } \\
\text { religious affiliation }\end{array}$ & $\begin{array}{l}\text { SCS was not associated with } \\
\text { religious affiliation in either sample } \\
\text { (male sex workers or non-male sex } \\
\text { workers). }\end{array}$ \\
\hline Efrati (2018a) & $\begin{array}{c}N=310 ; 11 \text { th and } 12 \text { th graders } \\
\text { Israel } \\
M=16.94, \mathrm{SD}=0.65\end{array}$ & $\begin{array}{c}59 \% \text { boys }(n=183) \text { and } 41 \% \text { girls } \\
(n=127) \\
95.8 \% \text { Native Israeli } \\
\text { Unreported }\end{array}$ & $\begin{array}{l}\text { I-CSB \& basic demographic } \\
\text { information regarding religiosity }\end{array}$ & $\begin{array}{l}\text { CSB is not significantly related to } \\
\text { religiosity in their model on the link } \\
\text { between attachment, temperament, } \\
\text { gender, and religious status to CSB } \\
\text { and psychopathology. } \\
\text { (continued) }\end{array}$ \\
\hline
\end{tabular}


Table 1. Continued

\begin{tabular}{|c|c|c|c|c|}
\hline Authors/Year & $\begin{array}{l}\text { Sample/Country } \\
\text { Age }(\text { Mean/SD) }\end{array}$ & $\begin{array}{c}\text { Gender/Race/ } \\
\text { Sexual Orientation }\end{array}$ & $\begin{array}{l}\text { CSB \& Religiosity/ } \\
\text { Spirituality Measure }\end{array}$ & Results \\
\hline Efrati (2018b) & $\begin{array}{c}N=274 ; 10 \text { th, } 11 \text { th, and } 12 \text { th } \\
\text { graders } \\
\text { Israel } \\
M=16.84, \mathrm{SD}=1.29\end{array}$ & $\begin{array}{c}47.8 \% \text { boys }(n=131) \text { and } 52.2 \% \\
\text { girls }(n=143) \\
\text { 93.1\% Native Israeli } \\
\text { Unreported }\end{array}$ & $\begin{array}{l}\text { I-CSB, Sensation of Shame of I- } \\
\text { CSB, \& basic demographic } \\
\text { information regarding religiosity }\end{array}$ & $\begin{array}{l}\text { Among secular people, higher CSB } \\
\text { was associated with higher levels of } \\
\text { autonomous help seeking. Among } \\
\text { religious people, CSB and } \\
\text { autonomous help seeking were not } \\
\text { linked in the model; however, } \\
\text { greater CSB was associated with } \\
\text { higher levels of avoidant help } \\
\text { seeking behaviors in religious } \\
\text { adolescents. }\end{array}$ \\
\hline $\begin{array}{l}\text { Grubbs Wilt, Exline and Pargament } \\
(2018 \text {, Study } 1)^{\mathrm{a}}\end{array}$ & $\begin{array}{c}\text { T1: } N=1,352 ; \mathrm{T} 2: N=265,146 \text { for } \\
\text { some analyses; undergraduates } \\
\text { US } \\
\text { Unreported }\end{array}$ & $\begin{array}{c}67.7 \% \text { men } \\
\text { 69.2\% White/Caucasian } \\
90.1 \% \text { heterosexual }\end{array}$ & $\begin{array}{c}\text { CPUI-9 \& aggregate score of RBSM } \\
\text { \& ARPM }\end{array}$ & $\begin{array}{c}\text { Found an association between the } \\
\text { CPUI- } 9 \text { and religiosity }(r=0.48, \\
P<0.005) \text {. There was also a large } \\
\text { correlation between religiosity and } \\
\text { moral disapproval }(r=0.71, P< \\
0.005) .\end{array}$ \\
\hline $\begin{array}{l}\text { Grubbs Wilt, Exline and Pargament } \\
\qquad(2018 \text {, Study 2) }\end{array}$ & $\begin{array}{c}\mathrm{T} 1: N=793 ; \mathrm{T} 2: N=360,176 \text { for } \\
\text { some analyses } \\
\text { US } \\
\text { Unreported }\end{array}$ & $\begin{array}{c}\text { T1: } 48.8 \% \text { men; T2: } 52 \% \text { men } \\
\text { 79.3\% White/Caucasian } \\
\text { 83.9\% heterosexual }\end{array}$ & $\begin{array}{c}\text { CPUI-9 \& aggregate score of RBSM } \\
\text { \& ARPM }\end{array}$ & $\begin{array}{c}\text { Found an association between the } \\
\text { CPUI-9 and religiosity }(r=0.36, \\
P<0.005) \text {. There was also a large } \\
\text { correlation between religiosity and } \\
\text { moral disapproval }(r=0.61, P< \\
0.005) .\end{array}$ \\
\hline $\begin{array}{l}\text { Grubbs Wilt, Exline, Pargament and } \\
\text { Kraus }(2018, \text { Study } 1)^{\mathrm{a}}\end{array}$ & $\begin{array}{c}\mathrm{T} 1: N=1,507 ; \text { at } \mathrm{T} 2: N=146 \\
\text { undergraduates } \\
\text { US } \\
M=19.3, \mathrm{SD}=2.2\end{array}$ & $\begin{array}{c}\text { T1: } 65.2 \% \text { men, } 34.5 \% \text { women, and } \\
0.3 \% \text { other } \\
\text { 90.1\% heterosexual } \\
\text { T2: } 67.2 \% \text { men } \\
\text { 83.9\% heterosexual } \\
\text { 69.2\% White/Caucasian }\end{array}$ & $\begin{array}{c}\text { CPUI-9 \& aggregate of RBSM \& } \\
\text { ARPM }\end{array}$ & $\begin{array}{l}\text { Found significant positive } \\
\text { associations between religiousness } \\
\text { and CPUI-9 scores }(r=0.48, P< \\
\text { 0.01). Religiousness at baseline was } \\
\text { correlated with CPUI-9 scores one } \\
\text { year later }(r=0.39, P<0.01) ; \\
\text { however, these findings were not } \\
\text { significant after controlling for } \\
\text { baseline beliefs about addiction. } \\
\text { There was a large positive } \\
\text { correlation between religiosity and } \\
\text { moral disapproval. }\end{array}$ \\
\hline $\begin{array}{l}\text { Grubbs Wilt, Exline, Pargament and } \\
\text { Kraus }(2018 \text {, Study } 2)^{\mathrm{a}}\end{array}$ & $\begin{array}{c}\text { T1: } N=782 ; \text { at T2: } N=211 \\
\qquad \begin{array}{c}\text { US } \\
M=32.6, \mathrm{SD}=10.3\end{array}\end{array}$ & $\begin{array}{c}\mathrm{T} 1: 48.8 \% \text { men, } 50.6 \% \text { women, and } \\
0.6 \% \text { other; } \mathrm{T} 2: 73.5 \% \text { men } \\
\text { 79.3\% White/Caucasian } \\
\text { 83.9\% heterosexual }\end{array}$ & $\begin{array}{c}\text { CPUI-9 \& aggregate of RBSM \& } \\
\text { ARPM }\end{array}$ & $\begin{array}{l}\text { Found significant positive } \\
\text { associations between religiousness } \\
\text { and CPUI-9 scores }(r=0.36, P< \\
\text { 0.01). There was a large positive } \\
\text { correlation between religiosity and } \\
\text { moral disapproval. } \\
\text { (continued) }\end{array}$ \\
\hline
\end{tabular}




\begin{tabular}{|c|c|c|c|c|}
\hline Authors/Year & $\begin{array}{l}\text { Sample/Country } \\
\text { Age }(\text { Mean/SD) }\end{array}$ & $\begin{array}{c}\text { Gender/Race/ } \\
\text { Sexual Orientation }\end{array}$ & $\begin{array}{l}\text { CSB \& Religiosity/ } \\
\text { Spirituality Measure }\end{array}$ & Results \\
\hline Grubbs, Grant, et al. (2018, Study 1) & $\begin{array}{c}N=829 \\
\text { Unreported } \\
M=33.3, \mathrm{SD}=9.4\end{array}$ & $\begin{array}{c}56.7 \% \text { men } \\
79 \% \text { White/Caucasian } \\
86 \% \text { heterosexual }\end{array}$ & $\begin{array}{l}\text { Single items specific to study } \\
\text { regarding PPU \& aggregate of } \\
\text { RBSM \& ARPM }\end{array}$ & $\begin{array}{l}\text { There was a small association } \\
\text { between religiosity the following } \\
\text { statements: "believe I am addicted } \\
\text { to internet pornography" ( } r= \\
0.149,95 \% \mathrm{CI}[0.079,0.217]) \text { and "I } \\
\text { would call myself an internet } \\
\text { pornography addict" }(r=0.089, \\
95 \% \mathrm{CI}[0.019,0.159]) .\end{array}$ \\
\hline Grubbs, Grant, et al. (2018, Study 3) & $\begin{array}{l}N=231 ; \text { undergraduates } \\
\text { US } \\
\quad M=19.3, \mathrm{SD}=1.8\end{array}$ & $\begin{array}{c}39.8 \% \text { men } \\
\text { 83\% White/Caucasian } \\
79 \% \text { heterosexual }\end{array}$ & $\begin{array}{l}\text { Single items specific to study } \\
\text { regarding PPU \& aggregate of } \\
\text { RBSM \& ARPM }\end{array}$ & $\begin{array}{l}\text { Religiosity was not associated with } \\
\text { the statements "I believe I am } \\
\text { addicted to internet pornography" } \\
\text { and "I would call myself an internet } \\
\text { pornography addict." }\end{array}$ \\
\hline Grubbs, Grant, et al. (2018, Study 4) & $\begin{array}{c}N=736 \\
\mathrm{US} \\
M=48, \mathrm{SD}=15.8\end{array}$ & $\begin{array}{c}58.1 \% \text { men } \\
75 \% \text { White/Caucasian } \\
\text { 90\% heterosexual }\end{array}$ & $\begin{array}{l}\text { Single items specific to study } \\
\text { regarding PPU \& the mean of single } \\
\text { items specific to study regarding } \\
\text { religiosity }\end{array}$ & $\begin{array}{l}\text { Religiosity was not associated with } \\
\text { the statements "I believe I am } \\
\text { addicted to internet pornography" } \\
\text { and "I would call myself an internet } \\
\text { pornography addict." }\end{array}$ \\
\hline $\begin{array}{l}\text { Kohut and Štulhofer } \\
\quad(2018 \text {, Study } 1)^{\mathrm{a}}\end{array}$ & $\begin{array}{c}\text { Rijeka Panel; } N=314 \\
\text { Croatia } \\
24.41 \%=15 \text { and under; } 73.23 \%= \\
16 ; 2.36 \% 17 \text { and over }\end{array}$ & $\begin{array}{l}100 \% \text { boys } \\
\text { Unreported } \\
\text { Unreported }\end{array}$ & $\begin{array}{l}\text { CPCS, Faith in God measure, \& } \\
\text { single item specific to study } \\
\text { regarding religiosity }\end{array}$ & $\begin{array}{l}\text { Religiosity was not associated with } \\
\text { any measure of pornography use or } \\
\text { the CPCS. }\end{array}$ \\
\hline $\begin{array}{l}\text { Kohut and Štulhofer } \\
\quad(2018 \text {, Study 2) }\end{array}$ & $\begin{array}{c}\text { Zagreb Panel; } N=197 \\
\text { Croatia } \\
1.90 \%=15 \text { and under; } 81.43 \%= \\
16 ; 16.67 \%=17 \text { and over }\end{array}$ & $\begin{array}{l}100 \% \text { boys } \\
\text { Unreported } \\
\text { Unreported }\end{array}$ & $\begin{array}{l}\text { CPCS, Faith in God measure, \& } \\
\text { single item specific to study } \\
\text { regarding religiosity }\end{array}$ & $\begin{array}{l}\text { The CPCS was not correlated with } \\
\text { Church attendance or personal } \\
\text { faith. }\end{array}$ \\
\hline Leonhardt et al. (2018) & $\begin{array}{c}N=686 \\
\mathrm{US} \\
M=28.54, \mathrm{SD}=7.85\end{array}$ & $\begin{array}{c}51 \% \text { men }(n=350) \text { and } 49 \% \\
\text { women }(n=336 \\
60 \% \text { White/Caucasian } \\
\text { Unreported }\end{array}$ & $\begin{array}{l}\text { SCS (Adapted scale) \& single } \\
\text { religiosity items specific to study }\end{array}$ & $\begin{array}{l}\text { Found a positive correlation ( } r= \\
0.29, P<0.05) \text { between religiousness } \\
\text { (dichotomous variable, low vs. high } \\
\text { religiousness) and perception of } \\
\text { addiction. }\end{array}$ \\
\hline
\end{tabular}


Table 1. Continued

\begin{tabular}{|c|c|c|c|c|}
\hline Authors/Year & $\begin{array}{l}\text { Sample/Country } \\
\text { Age }(\text { Mean/SD) }\end{array}$ & $\begin{array}{c}\text { Gender/Race/ } \\
\text { Sexual Orientation }\end{array}$ & $\begin{array}{l}\text { CSB \& Religiosity/ } \\
\text { Spirituality Measure }\end{array}$ & Results \\
\hline Zilberman et al. (2018) & $\begin{array}{l}\text { Clinical sample: } N=61 \\
\text { Control sample: } N=78 \\
\text { Israel } \\
\text { Unreported }\end{array}$ & $\begin{array}{c}93.4 \% \text { men }(n=57) \text { and } 6.5 \% \\
\text { women }(n=4) \\
\text { Unreported } \\
\text { Unreported }\end{array}$ & $\begin{array}{l}\text { I-CSB \& self-report measure of } \\
\text { religiosity }\end{array}$ & $\begin{array}{l}\text { Chi square test indicated } \\
\text { significantly different distribution of } \\
\text { religious, traditional, and non- } \\
\text { religious individuals across different } \\
\text { categories of addiction (alcohol, } \\
\text { drug, sex, gambling, and control) } \chi 2 \\
(8)=130.418 P<0.001 \text {. There was } \\
\text { a higher prevalence of religious } \\
\text { individuals in the sex addiction } \\
\text { group }(n=53) \text { compared to } \\
\text { traditional }(n=2) \text { and non- } \\
\text { religious }(n=6) .\end{array}$ \\
\hline Efrati (2019, Study 1) & $N=661$ & $\begin{array}{l}49.8 \% \text { boys }(n=329) \text { and } 50.2 \% \\
\text { girls }(n=332)\end{array}$ & $\begin{array}{c}\text { I-CSB \& basic demographic } \\
\text { information regarding religiosity }\end{array}$ & $\begin{array}{l}\text { Religious adolescents scored } \\
\text { significantly higher than secular }\end{array}$ \\
\hline & $\begin{array}{c}\text { Israel } \\
M=16.84, \mathrm{SD}=1.29\end{array}$ & $\begin{array}{l}\text { Unreported } \\
\text { Unreported }\end{array}$ & I CCD R b. J l & $\begin{array}{c}\text { adolescents on CSB }(\beta=0.84, P< \\
0.001) .\end{array}$ \\
\hline Efrati (2019, Study 2) & $\begin{array}{c}N=522 \\
\text { Israel } \\
M=16.84, \mathrm{SD}=1.29\end{array}$ & $\begin{array}{c}43.5 \% \text { boys }(n=227) \text { and } 56.5 \% \\
\text { girls }(n=295) \\
\text { Unreported } \\
\text { Unreported }\end{array}$ & $\begin{array}{c}\text { I-CSB \& basic demographic } \\
\text { information regarding religiosity b }\end{array}$ & $\begin{array}{l}\text { Religious adolescents were higher } \\
\text { than their secular adolescents on } \\
\text { CSB }(\beta=0.75, P=0.002)\end{array}$ \\
\hline Efrati (2019, Study 3) & $\begin{array}{c}N=317 \\
\text { Israel } \\
M=17.84, \mathrm{SD}=4.23\end{array}$ & $\begin{array}{c}49.5 \% \text { boys }(n=157) \text { and } 50.5 \% \\
\text { girls }(n=160) \\
\text { Unreported } \\
\text { Unreported }\end{array}$ & $\begin{array}{l}\text { I-CSB \& basic demographic } \\
\text { information regarding religiosity }\end{array}$ & $\begin{array}{l}\text { Sexual thought suppression } \\
\text { mediated the association between } \\
\text { religiosity and CSB. }\end{array}$ \\
\hline Grubbs, Kraus, et al. (2019) & $\begin{array}{c}N=2,075 \text { Analyzed: } N=1,461 \\
\text { US } \\
M=44.8, \mathrm{SD}=16.7\end{array}$ & $\begin{array}{c}59 \% \text { men } \\
\text { 74\% White/Caucasian } \\
\text { Unreported }\end{array}$ & $\begin{array}{l}\text { CPUI-9 (adapted version Part 1) \& } \\
\text { the mean of single items specific to } \\
\text { study regarding religiosity }\end{array}$ & $\begin{array}{l}\text { Religiosity was not correlated with } \\
\text { frequency of pornography use or } \\
\text { amount consumed per day, but was } \\
\text { correlated with each of the three } \\
\text { subscale representative items }(r= \\
0.205, P<0.001 ; r=0.194, P< \\
0.001 ; r=0.317, P<0.001) \text {, with the } \\
\text { three item self-perceived addiction } \\
\text { measure }(r=0.283, P<0.001) \text {, and } \\
\text { the moral incongruence measure } \\
(r=0.447, P<0.001) \text {. } \\
\text { (continued) }\end{array}$ \\
\hline
\end{tabular}




\begin{tabular}{|c|c|c|c|c|}
\hline Authors/Year & $\begin{array}{l}\text { Sample/Country } \\
\text { Age }(\text { Mean/SD) }\end{array}$ & $\begin{array}{c}\text { Gender/Race/ } \\
\text { Sexual Orientation }\end{array}$ & $\begin{array}{l}\text { CSB \& Religiosity/ } \\
\text { Spirituality Measure }\end{array}$ & Results \\
\hline Maddock et al. (2019) ${ }^{\mathbf{a}}$ & $\begin{array}{c}\text { T1 } N=320 \text { Time } 2(3 \text { months }) N= \\
175 \text { Time } 3(6 \text { months } N=163 \\
\text { Unreported } \\
M=36.26, \mathrm{SD}=10.18\end{array}$ & $\begin{array}{c}54.8 \% \text { men and } 45.3 \% \text { women } \\
\text { 74.7\% White/Caucasian } \\
86 \% \text { heterosexual }\end{array}$ & $\begin{array}{c}\text { PPUS, CSB measure created } \\
\text { specifically for study, \& single items } \\
\text { specific to study regarding } \\
\text { religiosity }\end{array}$ & $\begin{array}{l}\text { Religiosity at baseline did not } \\
\text { predict excessive or problematic } \\
\text { pornography use at } 3 \text { months, and } \\
\text { the interaction of religiosity and } \\
\text { frequency of pornography use at } \\
\text { baseline also did not predict } \\
\text { excessive or compulsive use at } 3 \\
\text { months. More religious people who } \\
\text { viewed pornography were about } \\
\text { equally as likely to view their use as } \\
\text { excessive or compulsive as less } \\
\text { religious people. }\end{array}$ \\
\hline Rosmarin and Pirutinsky (2019) ${ }^{\mathbf{a}}$ & $\begin{array}{c}N=94 \\
\mathrm{US} \\
M=40.15, \mathrm{SD}=16.11\end{array}$ & $\begin{array}{l}100 \% \text { men } \\
\text { Unreported } \\
\text { Unreported }\end{array}$ & $\begin{array}{l}\text { Clinical interview, DRI, JCOPE, \& } \\
\text { single items specific to study } \\
\text { regarding religiosity }\end{array}$ & $\begin{array}{l}\text { Religious struggles subscale of } \\
\text { JCOPE was only positively } \\
\text { correlated with problematic sexual } \\
\text { behaviors among individual who } \\
\text { were raised orthodox, whether they } \\
\text { were currently still orthodox ( } r= \\
0.58, P<0.01) \text { or were no longer } \\
\text { orthodox }(r=0.47, P<0.05) \text {. No } \\
\text { other variables were correlated for } \\
\text { these two groups, and there was no } \\
\text { association between religiosity and } \\
\text { sexual behavior for these who were } \\
\text { not raised orthodox. }\end{array}$ \\
\hline Borgogna et al. (2020) & $\begin{array}{c}N=224 \\
\mathrm{US} \\
M=19.63, \mathrm{SD}=66.22\end{array}$ & $\begin{array}{c}100 \% \text { men } \\
72 \% \text { White/Caucasian } \\
100 \% \text { heterosexual }\end{array}$ & $\begin{array}{l}\text { CPUI-9, pornography viewing } \\
\text { frequency, DRS, "Fear of Sin" } \\
\text { subscale from the PIOS, \& single } \\
\text { items specific to study regarding } \\
\text { religiosity }\end{array}$ & $\begin{array}{l}\text { Found that the CPUI- } 9 \text { correlated } \\
\text { with the DRS ( } r=0.17, P<0.01) \text {, } \\
\text { the "Fear of } \operatorname{Sin} \text { " subscale of } \\
\text { scrupulosity ( } r=0.5, P<0.001 \text { ), } \\
\text { spirituality ("How spiritual are } \\
\text { you?"; } r=0.14, P<0.05 \text { ), and } \\
\text { religious behavior (frequency of } \\
\text { religious activities, such as prayer; } \\
r=0.31, P<0.001 \text { ). } \\
\text { (continued) }\end{array}$ \\
\hline
\end{tabular}


Table 1. Continued

\begin{tabular}{|c|c|c|c|c|}
\hline Authors/Year & $\begin{array}{l}\text { Sample/Country } \\
\text { Age }(\text { Mean/SD) }\end{array}$ & $\begin{array}{c}\text { Gender/Race/ } \\
\text { Sexual Orientation }\end{array}$ & $\begin{array}{l}\text { CSB \& Religiosity/ } \\
\text { Spirituality Measure }\end{array}$ & Results \\
\hline $\begin{array}{l}\text { Grubbs, Kraus, et al. } \\
\quad(2020 \text {, Sample 1) }\end{array}$ & $\begin{array}{c}N=467 ; \text { undergraduates } \\
\text { US } \\
M=19.32, \mathrm{SD}=2.45\end{array}$ & $\begin{array}{l}38.5 \% \text { men } \\
\text { 82\% White/Caucasian } \\
\text { Unreported }\end{array}$ & $\begin{array}{c}\text { CPUI-4 \& aggregate score of RBSM } \\
\text { \& ARPM }\end{array}$ & $\begin{array}{c}\text { Religiosity was found to be } \\
\text { associated with both self-reported } \\
\text { problems }(r=0.183, P<0.005) \text { and } \\
\text { moral disapproval }(r=0.517, P< \\
0.005) .\end{array}$ \\
\hline $\begin{array}{l}\text { Grubbs, Kraus, et al. } \\
\quad(2020 \text {, Sample 2) }\end{array}$ & $\begin{array}{c}N=739 \\
\mathrm{US} \\
M=47.9, \mathrm{SD}=15.81\end{array}$ & $\begin{array}{c}58 \% \text { men } \\
74.7 \% \text { White/Caucasian } \\
\text { Unreported }\end{array}$ & $\begin{array}{l}\text { CPUI- } 4 \text { \& single items specific to } \\
\text { study regarding religiosity }\end{array}$ & $\begin{array}{l}\text { Religiosity was found to be } \\
\text { positively associated with self- } \\
\text { reported problems }(r=0.074, P< \\
0.05) \text { and moral disapproval }(r= \\
0.517, P<0.005)\end{array}$ \\
\hline $\begin{array}{l}\text { Grubbs, Kraus, et al. } \\
\quad(2020 \text {, Sample 3) }\end{array}$ & $\begin{array}{c}N=1,461 \\
\mathrm{US} \\
M=45.51, \mathrm{SD}=16.60\end{array}$ & $\begin{array}{l}59 \% \text { men } \\
74 \% \text { White/Caucasian } \\
\text { Unreported }\end{array}$ & $\begin{array}{l}\text { BPS \& single items specific to study } \\
\text { regarding religiosity }\end{array}$ & $\begin{array}{l}\text { Religiosity was found to be } \\
\text { positively associated with self- } \\
\text { reported problems }(r=0.144, P< \\
0.005) \text { and moral disapproval }(r= \\
0.291, P<0.005)\end{array}$ \\
\hline $\begin{array}{l}\text { Grubbs, Kraus, et al. } \\
\quad(2020, \text { Sample } 4)^{\mathrm{a}}\end{array}$ & $\begin{array}{c}\text { T1 } N=850 \text { Time } 2(4 \text { month }) N= \\
512 \text { Time } 3(8 \text { month }) N=477 \\
\text { Time } 4(12 \text { month }) N=428 \\
\text { US } \\
M=33.98, S D=9.87\end{array}$ & $\begin{array}{c}52.3 \% \text { men } \\
\text { 78.9\% White/Caucasian } \\
\text { Unreported }\end{array}$ & $\begin{array}{l}\text { CPUI-4 \& aggregate score of RBSM } \\
\text { \& ARPM }\end{array}$ & $\begin{array}{c}\text { Religiosity was found to be } \\
\text { positively associated with moral } \\
\text { disapproval at all phases (T1: } r= \\
0.373, P<0.005 \text {; T2: } r=0.371, P< \\
0.005 \text {; T3: } r=0.4, P<0.005 \text {; T4: } \\
r=0.366, P<0.005 \text { ) and with self- } \\
\text { reported problems at all phases (T1: } \\
r=0.203, P<0.005 \text {; T2: } r=0.238, \\
P<0.005 \text {; T3: } r=0.2, P<0.005 \text {; T4: } \\
r=0.138, P<0.005) .\end{array}$ \\
\hline Grubbs, Lee, et al. (2020) & $\begin{array}{c}N=1,424 \\
\mathrm{US} \\
M=43.92, \mathrm{SD}=16.74\end{array}$ & $\begin{array}{c}66.4 \% \text { men } \\
62.5 \% \text { White/Caucasian } \\
\text { Unreported }\end{array}$ & $\begin{array}{l}\text { CPUI-4, BPS, \& Pew Research } \\
\text { Center Survey items }\end{array}$ & $\begin{array}{l}\text { Religiosity was found to be } \\
\text { positively associated with the BPS } \\
\text { mean score }(r=0.262,95 \% \text { CI } \\
[0.211,0.311]) \text { and the CPUI- } 4(r= \\
0.158,95 \% \text { CI }[0.105,0.209]) \text {. } \\
\text { Religiousness acted as a moderator } \\
\text { between pornography use and self- } \\
\text { reported addiction, such that } \\
\text { pornography use was more strongly } \\
\text { related to self-reported addiction at } \\
\text { higher levels of religiosity. } \\
\text { (continued) }\end{array}$ \\
\hline
\end{tabular}




\begin{tabular}{|c|c|c|c|}
\hline Authors/Year & $\begin{array}{l}\text { Sample/Country } \\
\text { Age (Mean/SD) }\end{array}$ & $\begin{array}{c}\text { Gender/Race/ } \\
\text { Sexual Orientation }\end{array}$ & $\begin{array}{l}\text { CSB \& Religiosity/ } \\
\text { Spirituality Measure }\end{array}$ \\
\hline \multirow[t]{2}{*}{ Lewczuk et al. (2020) } & $N=885$ & $\begin{array}{c}55.1 \% \text { men }(n=485) \text { and } 44.9 \% \\
\text { women }(n=395)\end{array}$ & $\begin{array}{l}\text { BPS, HBI-19, CPUI-9 (adapted } \\
\text { version), religious affiliation, \& }\end{array}$ \\
\hline & $\begin{array}{c}\text { Poland } \\
M=43.69, \mathrm{SD}=14.06\end{array}$ & $\begin{array}{l}\text { Unreported } \\
\text { Unreported }\end{array}$ & $\begin{array}{l}\text { single items specific to study } \\
\text { regarding religiosity }\end{array}$ \\
\hline
\end{tabular}

Zimmer and Imhoff (2020)

$$
\begin{gathered}
N=1,063 \\
\text { Multinational } \\
M=26.86, \mathrm{SD}=6.79
\end{gathered}
$$

$100 \%$ men

Unreported

Unreported

\section{De Jong and Cook (2021)}

\section{$N=646$ participants identified as theist}

\section{$63 \%$ men}

78.6\% White/Caucasian, 77.7\% heterosexual
HBI, TSO, \& religious affiliation

CPUI-9, DUREL, \& PIOS-R
Religiosity was not correlated with self-perceived pornography addiction but was positively associated with problematic pornography use $(r=0.11, P<$ $0.001)$, moral disapproval of pornography $(r=0.44, P<0.001)$ moral incongruence related distress $(r=0.22, P<0.001)$. The path analyses indicated that religiosity contributes to the above

pornography constructs, which then contribute to problematic

$$
\text { pornography use. }
$$

Did not find a correlation between participants identifying as Christian and HBI-19 scores; however, there was a small correlation between the HBI-19 and religious affiliation $(r=$ $0.17, P<0.005)$, as well as annual frequency of attending Church service $(r=0.17, P<0.005)$. The dyscontrol subscale of the HBI-19 correlated with Christian affiliation $(r=0.14, P<0.005)$, other religious affiliation $(r=0.20, P<0.005)$, and annual frequency of attending Church service $(r=0.25, P<$

$$
0.005) \text {. }
$$

Experimental manipulation of religious priming did not have an indirect effect via shame on selfperceived perceived pornography addiction. Exploratory analyses revealed an indirect effect of religious primes via shame on selfperceived pornography addiction among individuals high on both organizational religiosity and

various obsessive compulsive disorder compulsivity subscales. 
Table 1. Continued

\begin{tabular}{|c|c|c|c|c|}
\hline Authors/Year & $\begin{array}{l}\text { Sample/Country } \\
\text { Age (Mean/SD) }\end{array}$ & $\begin{array}{c}\text { Gender/Race/ } \\
\text { Sexual Orientation }\end{array}$ & $\begin{array}{l}\text { CSB \& Religiosity/ } \\
\text { Spirituality Measure }\end{array}$ & Results \\
\hline $\begin{array}{l}\text { Efrati and Amichai-Hamburger } \\
\text { (2021) Study } 2\end{array}$ & $\begin{array}{c}N=713 \\
\text { Adolescents age } 14-18 \\
\text { Israel } \\
M=16.71, \mathrm{SD}=1.17\end{array}$ & $\begin{array}{l}53.7 \% \text { boys } \\
\text { Not reported } \\
\text { Not reported }\end{array}$ & $\begin{array}{l}\text { I-CSB and religious status: religious } \\
\text { or secular }{ }^{\mathrm{b}}\end{array}$ & $\begin{array}{l}\text { Results indicated that adolescents } \\
\text { who only engaged in online sexual } \\
\text { activities had significantly higher } \\
\text { percentage of clinical CSB and were } \\
\text { more likely to be religious. }\end{array}$ \\
\hline Hotchkiss (2021) & $\begin{array}{c}N=464 \\
\mathrm{US} \\
M=33.4, \mathrm{SD}=16.4\end{array}$ & $\begin{array}{c}95.4 \% \text { men } \\
\text { 77.8\% White/Caucasian, Not } \\
\text { reported }\end{array}$ & SCS and RSSS & $\begin{array}{l}\text { Significant difference between } \\
\text { religious }(M=65,66 ; \mathrm{SD}=21.31) \\
\text { and non-religious }(M=53,08, \mathrm{SD} \\
=22.40) \text { scores on the RSSS }(F= \\
15.09, P=0.001) .\end{array}$ \\
\hline Leonhardt et al. (2021) & $\begin{array}{c}N=1,421 \\
\mathrm{US} \\
M=34.12, \mathrm{SD}=10.46\end{array}$ & $\begin{array}{c}55.7 \% \text { men } \\
76 \% \text { White } \\
2.4 \% \text { reported some same-sex } \\
\text { attraction }\end{array}$ & $\begin{array}{c}\text { Perceived Compulsivity Subscale of } \\
\text { the CPUI-9 and } 3 \text { items from } \\
\text { RELATE questionnaire assessing } \\
\text { religiosity }\end{array}$ & $\begin{array}{c}\text { Religiosity was not associated with } \\
\text { self-perceived problematic } \\
\text { pornography use for men or } \\
\text { women. }\end{array}$ \\
\hline Lewczuk et al. (2021) & $\begin{array}{c}N=1,036 \\
\text { Poland } \\
M=43.28, \mathrm{SD}=14.21\end{array}$ & $\begin{array}{l}49 \% \text { men } \\
\text { Not reported - all Polish } \\
\text { Not reported }\end{array}$ & $\begin{array}{l}\text { Three items from the CPUI-9; three } \\
\text { items measuring various aspects of } \\
\text { religiosity }\end{array}$ & $\begin{array}{c}\text { Moral incongruence }(\beta=0.20, P< \\
0.001) \text { and religiosity }(\beta=0.08, P< \\
0.05) \text { were significant predictors of } \\
\text { self-perceived pornography } \\
\text { addiction. }\end{array}$ \\
\hline Rousseau et al. $(2021)^{\mathrm{a}}$ & $\begin{array}{l}\qquad N=337 \\
\text { Croatia } \\
\text { Not reported; all were high school } \\
\text { sophomores at time of recruitment }\end{array}$ & $\begin{array}{l}\text { All men } \\
\text { Not reported - all Croatian } \\
\text { Not reported }\end{array}$ & $\begin{array}{l}\text { Compulsive Pornography } \\
\text { Consumption scale; } 4 \text {-item measure } \\
\text { of religious faith used in previous } \\
\text { research in Croatia }\end{array}$ & $\begin{array}{c}\text { Greater porn use at baseline and } \\
\text { greater increase in porn use over } \\
\text { time was related to increased PPU } \\
\text { at T6. This relationship was } \\
\text { moderated by religiosity, such that } \\
\text { there was a stronger relationship } \\
\text { between pornography frequency/ } \\
\text { increase and PPU for more religious } \\
\text { individuals. Religiosity was not } \\
\text { correlated with PPU scores at T5/ } \\
\text { T6. }\end{array}$ \\
\hline
\end{tabular}

Note. CSB measure abbreviations include: The Male Sexual Addiction Screening Test (G-SAST), Hypersexual Behavioral Consequences Scale (HBCS), Hypersexual Disorder Questionnaire (HDQ), Hypersexual Behavior Inventory (HBI-19), Cyber Pornography Use Inventory-9 (CPUI-9), Cyber Pornography Use Inventory-4 (CPUI-4), Sexual Compulsivity Scale (SCS), Brief Pornography Screener (BPS), Compulsive Pornography Consumption Scale (CPCS), Compulsive Sexual Behavior Inventory (CSBI), Hypersexual Disorder Screening Inventory (HDSI), Sexual Addiction Screening Test (SAST), Sexual Addiction Screening Test - Revised (SAST-R), Individual-Based Compulsive Sexual Behavior Scale (I-CSB), and “Total Sexual Outlet" (TSO) measure. Religiosity and spirituality measure abbreviations include: Systems of Belief Inventory (SBI-15R), Spiritual Well-Being Scale (SWBS), Dimensions of Religiosity Scale (DRS), Religious

Commitment Inventory (RCI), Brief Religious Coping Scale (Brief RCOPE), Religious and Spiritual Struggles Scale (RSS), Jewish Religious Coping Scale (JCOPE), Spiritual Struggle Scale (SSS), Duke University Religion Index (DUREL), The Penn Inventory of Scrupulosity (PIOS), Religious Belief Salience measure (RBSM), and Adapted Religious Participation Measure (ARPM).

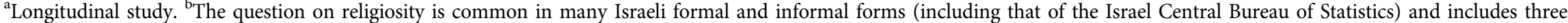

classifications: secular, religious (Orthodox) and ultra-Orthodox. 
(Joseph \& Diduca, 2007). No studies reported negative or only non-significant relationships.

\section{Relationship between the $\mathrm{HBI}-19$ and religiosity}

The HBI is a 19-item scale that assesses hypersexuality via three subscales: coping, control, and consequences (Reid et al., 2011). Seven studies utilized the HBI-19, though one study did not report associations between the HBI-19 and religiosity variables (Lewczuk et al., 2020). Three studies found no relationship between the HBI-19 and measures of religiosity, including religious belief (e.g., belief vs. no belief; Dhuffar \& Griffiths, 2014), religious commitment (Reid et al., 2016), and identifying as Christian (Zimmer \& Imhoff, 2020). Four studies found a positive relationship between the HBI-19 and measures of religiosity or spirituality, including: negative religious coping (Giordano \& Cecil, 2014), religious/spiritual struggles (Griffin et al., 2016; Hook et al., 2015), religious affiliation, and attending Church services (Zimmer \& Imhoff, 2020). Giordano and Cecil (2014) also found that spiritual purpose and meaning was negatively associated with the HBI-19.

\section{Relationship between the I-CSB and religiosity}

The I-CSB is a 24-item scale that measures individual-based compulsive sexual behavior via four dimensions: unwanted consequences, lack of control, negative affect, and affect regulation (Efrati \& Mikulancer, 2018). Seven studies utilized the I-CSB. Of these, four reported a positive association between self-identification as religious and scores on the ICSB (Efrati, 2018b, 2019), with three of these studies being reported in Efrati (2019). One study reported a non-significant relationship between these constructs in their model on the link between attachment, temperament, gender, and religious status to CSB and psychopathology (Efrati, 2018a). Two studies only reported results of difference tests. One reported that adolescents engaging in online-only sexual activities, compared to offline and online sexual activities, were more likely to be religious and have clinically significant CSB (Efrati \& Amichai-Hamburger, 2021). The other study reported that religious individuals were significantly more likely to be in the CSB group than nonreligious persons (Zilberman et al., 2018). No studies reported a negative relationship between the I-CSB and religiosity or spirituality.

\section{DISCUSSION}

Research on CSB, religiosity, and spirituality has spiked in recent years and continues to gain relevance with the recent inclusion of CSBD in the ICD-11. Most studies in the literature use cross-sectional samples primarily composed of heterosexual White men and women from the United States. Research has focused on identifying associations between religiosity, moral incongruence, and PPU to explore how moral and religious conflict might complicate the assessment and treatment of possible manifestations of CSBD. Less research has focused on other possible areas of investigation, such as the association between spirituality and CSB or the possibility of certain religiosity constructs being inversely correlated with CSB (e.g., positive religious coping).

\section{Demographic representation in the literature}

A systematic review from Grubbs, Hoagland, et al. (2020) examined CSB research generally and provided an extensive demographic summary of studies in the field. In many respects, the demographic results in the present review are similar; however, there are also notable differences. In terms of similarities, the present review found that most studies have sampled a wide age range, with a smaller but substantial portion examining college students and adolescents. This result is similar to the broader CSB literature (Grubbs, Hoagland, et al., 2020). Notably, there was an absence of studies on CSB in older adults in the literate altogether.

In terms of gender, Grubbs, Hoagland, et al. (2020) reported that nearly half of all studies on CSB used exclusively male samples. Interestingly, only around $25 \%$ of studies examined men exclusively in the present review, indicating that an explicit focus on men in research on CSB religiosity, and spirituality is not as skewed as it is in the broader CSB research. Diverse gender identities remain an understudied population throughout the CSB literature. Grubbs, Hoagland, et al. (2020) also found that nearly $34 \%$ of studies did not report data on sexual orientation. In the present review, nearly half of the studies did not report sexual orientation demographic information. Further, relatively equal numbers of studies focused on heterosexual and MSM samples in the present review, whereas in Grubbs, Hoagland, et al. (2020) a greater proportion of studies examined CSB in LGB or MSM populations than in heterosexual samples.

In the present review, there was an overrepresentation of White individuals. Of the studies reporting data on race/ ethnicity, nearly $90 \%$ had White participants as the largest sampled group and these majorities were often substantial (above $75 \%$ of the sample). Grubbs, Hoagland, et al. (2020) did not provide information on this variable; however, there is a clear need to expand research on the CSB religiosity link to diverse racial/ethnic groups. Finally, most studies were conducted in Western, Educated, Industrialized, Rich, and Democratic (WEIRD) countries, particularly the US. These findings reflect the historical focus of sex research on WEIRD populations (Klein et al., 2021); however, it is notable that several studies were conducted in other countries, such as Croatia and Israel. The lack of diverse demographic data hinders the generalizability of research conclusions. Future research should consider the relationship between CSB and religiosity or spirituality among diverse populations.

\section{Measurement of CSB, religiosity, and spirituality}

This review noted considerable diversity in the assessment tools used to measure CSB. Only recently has the ICD-11 proposed criteria for a unified definition of CSBD, and thus, much of the literature is not consistent in the assessment of this syndrome. Religiosity and spirituality measures have 
also been diverse in their assessment of various constructs, with studies in the present review using a wide variety of validated and adapted measures or non-validated items.

Although there was significant diversity in the constructs assessed, general themes can be identified across measurement instruments. Measures of problematic pornography use, such as the CPUI-9, were the most common in the literature. This focus is warranted, given that most individuals who seek treatment for CSB struggle with PPU and compulsive masturbation (Grubbs, Kraus, et al., 2020; Reid et al., 2012). One explanation for this finding is that pornography and masturbation are both more accessible and less financially and socially costly than other sexual behaviors, such as hiring a sex worker or having a casual sexual partner (Grubbs, Kraus, et al., 2020; Grubbs, Perry, et al., 2019; Perry, 2019; Prause, 2019). For these reasons, the focus on PPU in the literature is quite appropriate.

Religiosity measures were far more common than measures of spirituality. Additionally, most measures of spirituality also assessed religiosity. The relationship between CSB and spirituality as a standalone construct has rarely been considered. To the extent that spirituality is distinct from religiosity, there may be novel relationships between this construct and CSB. The diverse array of religiosity constructs assessed by researchers has positive and negative aspects. Religiosity is a multifaceted and complex human experience, and several separate measures of religiosity are needed to capture this diversity. However, assessment of specific religiosity constructs across the literature was often inconsistent, with several studies using different measures of the same religiosity constructs, such as religious participation. Most notably, a large portion of studies described assessing general aspects of religiosity with one to three unvalidated items that often differed across studies. The inconsistent measurement of religiosity makes comparison across studies difficult. Researchers should continue exploring a diverse array of religiosity constructs when appropriate, but also measure religiosity in a more consistent manner.

In terms of religiosity measures, there was a large focus on religious participation and belief salience. Much of the present literature tends to focus on distinguishing perceived PPU from actual PPU (Grubbs, Perry, et al., 2019). These findings inform accurate assessment and treatment decisionmaking in clinical contexts; however, a relative lack of research on religious/spiritual struggles (Grubbs et al., 2017), religious coping (Giordano \& Cecil, 2014), and other relevant facets precludes understanding of distress arising from moral incongruence. Both accurate identification of CSB and relevant knowledge of distress due to moral incongruence (Lewczuk et al., 2020) will be necessary to be help individuals that do and do not meet CSBD criteria.

\section{The relationship between CSB, religiosity, and spirituality}

In general, the present review found that most studies reported a small to moderate positive relationship between
CSB and religiosity. However, there were also many nonsignificant relationships reported (Kohut \& Stulhofer, 2018; Reid et al., 2016; Skegg et al., 2010), as well as many associations that were very weak (Grubbs, Grant, et al., 2018; Grubbs, Kraus, et al., 2020; Lewczuk et al., 2020). The variety of measurement tools used, and constructs assessed across the literature, makes it difficult to draw more specific conclusions about the relationships between CSB and religiosity or spirituality. Divergent findings in the literature may be explained, in part, by the diverse measurement choices of researchers, as different aspects of CSB, religiosity, and spirituality are bound to have unique relationships with each other.

There are several notable considerations that may contribute to more consistent identification of a relationship between CSB and religiosity or spirituality. One of the most well-studied relationships in the literate is the association between PPU and an aggregate measure of belief salience and religious participation, which, as noted in the metaanalysis by Grubbs, Perry, et al. (2019), have consistently been positively associated. This relationship is strongly mediated by moral incongruence, with this path accounting for a large portion of the variance. Notably, recent research indicates that $\mathrm{MI}$ is better conceptualized as an interactive effect of pornography use and moral disapproval of pornography (Grubbs, Kraus, et al., 2020; Grubbs, Lee, et al., 2020). These studies report that moral disapproval moderates the relationship between pornography use and PPU such that pornography use is more strongly related to PPU at higher levels of moral disapproval.

These considerations are especially important in evaluation of the literature because many studies identified in the present review did not consider the possible mediating or moderating role of moral incongruence. Therefore, it stands to reason, that many of the small to moderate associations identified in the present review are due to the absence of these variables.

In addition to potential mediators and moderators, it was noted that the relationship between CSB and religiosity or spirituality differed based on the specific CSB construct that was assessed. For instance, identification of a direct association between religiosity and CSB was more consistent when studies examined problematic pornography use (most typically using the CPUI-9) instead of other forms of CSB. For instance, in the present study, associations between the HBI19 and religiosity and spirituality constructs did not produce as many significant relationships as did the studies using measures of problematic pornography use, such as the CPUI-9. PPU may be particularly salient for religious participants compared to other manifestations of CSB, as pornography may be the most easily accessible sexual medium with the lowest possible consequence (Grubbs, Kraus, et al., 2020). In addition, some studies found that only certain subscales of CSB measures (such as the CPUI-9) correlated with religiosity variables. For example, in study 2 of Grubbs, Exline, et al. (2015), the authors reported that two subscales of the CPUI-9 (perceived compulsivity and access efforts) were not directly correlated with religiosity; 
however, the access efforts and the overall CPUI-9 scores exhibited positive associations with religiosity. Thus, specific CSB sub-constructs may produce more consistent associations than others. Studies examining associations between $\mathrm{CSB}$, and religiosity longitudinally were similarly mixed. Some studies identified relationships between these constructs (Grubbs et al., 2017; Grubbs, Kraus, et al., 2020). However, several others did not identify a relationship between religiosity and CSB (Grubbs, Wilt, Exline, Pargament, \& Kraus, 2018; Kohut \& Štulhofer, 2018; Maddock et al., 2019).

Overall, the trends noted in the present review suggest that a relationship exists between religiosity and PPU, particularly in consideration of moral disapproval and moral incongruence. However, as described, there have been notable instances where direct relationships between religiousness and CSB have either not been replicated or have received partial support. Additionally, when a direct relationship between religiosity and PPU has been identified, these relationships are often quite small (Grubbs, Grant, et al., 2018; Grubbs, Perry, et al., 2019; Lewczuk et al., 2020; Volk et al., 2016). As indicated by the present review, other compulsive sexual behaviors besides PPU have received much less research attention. Although some research suggests an association between other forms of CSB and religiosity (Hook et al., 2015), a developed body of literature consistently demonstrating these relationships has yet to develop.

\section{Assessment and treatment considerations}

An inherent risk in CSB assessment is the conflation of this construct with distress arising from sexual behavior that conflicts with one's religious/spiritual or cultural values. As discussed in the present review, religiosity exhibits a strong association with PPU, via moral disapproval and moral incongruence, as well as a smaller to moderate direct association with CSB more broadly. This body of research adds support to the criteria in the ICD-11 that rules out diagnoses of CSBD when distress is entirely due to moral or cultural conflict with one's own sexual behavior, as religiosity may produce self-perception of sexual problems. Although CSB and sexual problems due to moral incongruence may appear similar topographically, each call for a distinct treatment approach, which can only be appropriately administered with accurate assessment of presenting symptomatology.

\section{Recommendations for future research}

In general, there is a lack of methodological rigor in this research area. Most studies examined the association between CSB and religiosity or spirituality using crosssectional surveys of convenience samples, with only a handful using longitudinal approaches. In addition, the use of convenience samples has resulted in an underrepresentation of diversity in age, sexual orientation, gender identity, and racial/ethnic identity. Future research should utilize more sophisticated research designs and expand sampling methods to include more representative samples, as well as samples targeting populations of interest. In addition to improving on methodology, future research should focus on consistency and validity of assessment. The wide variety of $\mathrm{CSB}$, religiosity, and spirituality measures used in the literature makes it difficult to draw meaningful conclusions. If future research focuses its attention on a select number of well-validated measures of CSB, religiosity, and spirituality, then researchers will be able to draw more substantive conclusions.

Finally, consistent with research on moral incongruence and the related rule-out criterion for CSBD in the ICD-11, future research on CSB assessment should account for the possible confounding influence of religiosity. Spirituality may also have a similar confounding effect as religiosity and should be given further consideration in the literature. If future research finds robust evidence for a relationship between CSB and religiosity or spirituality, clinical assessment tools for CSBD may include questions designed to measure clinically relevant aspects of these constructs, such as moral incongruence, that could have implications for diagnosis or treatment approach. Strong evidence has already been provided for the importance of assessing moral incongruence when evaluating PPU (Grubbs, Perry, et al., 2019). However, establishment of this relationship for other forms of CSB remains to be seen. Research on moral incongruence should be expanded to non-religious communities and other diverse populations. Individuals may not be religious, but still disapprove of their sexual behavior, which may complicate accurate assessment of CSBD. This possibility has yet to be investigated. Diverse groups may also have unique assessment considerations surrounding moral incongruence. For instance, sexually diverse individuals may experience MI because they disapprove of pornography use and the sexually diverse content of the pornography (e.g., gay sex). This possibility should be given additional research and clinical attention. Future research should therefore focus on developing clinically useful assessment tools that are considerate of diversity, evaluate all manifestations of CSB, and take into account clinically-relevant religiosity factors.

\section{Limitations}

A limitation of the present review is the absence of search terms involving the concept of moral incongruence or moral disapproval, given that much of the literature has examined these constructs in relation to religiosity and CSB. Although many of the articles in the present review examined moral disapproval and moral incongruence, the search terms in the present analysis did not allow for systematic evaluation of this relationship. Another limitation is that the present review only searched two databases and Google scholar, possibly hindering the identification of articles meeting inclusion criteria. In terms of demographic information, the present review did not collect data on the religious affiliations of participants reported in studies. Although other researchers (Grubbs, Perry, et al., 2019) have described that most studies have predominantly Christian samples, a systematic evaluation of all studies in the field would help clarify understanding of the associations between religiosity 
and CSB among other religious groups. Finally, some studies in the present review did not identify the association between CSB and religiosity as the focus of their study. Thus, the measurement approaches used in multiple studies were often not optimal for identifying the association between CSB and religiosity or spirituality.

\section{CONCLUSION}

Although there has been swift growth of research examining $\mathrm{CSB}$, religiosity, and spirituality over the past five years, developments are hampered by a lack of high-quality research design and under-representative sampling. Within this timeframe, a model of pornography problems due to moral incongruence has emerged highlighting the mediating or moderating variables that connect religiosity to PPU. Regarding a direct relationship between these constructs, the present review identified somewhat consistent small to moderate associations between CSB and religiosity or spirituality. However, methodological limitations of the literature prevented further interpretation. These findings should inform instrument development, assessment, and treatment approaches to avoid misdiagnosis of CSBD. Future research should advance the field by examining the association between CSB and religiosity or spirituality using more advanced methodological approaches, obtaining more representative samples, considering forms of CSB beyond PPU, and utilizing valid assessment tools consistently across studies.

Funding information: No financial support was received for this study.

Conflict of interest: The authors report no financial or other relationship relevant to the subject of this article.

Author's contribution: TLJ conceived of the presented idea, conducted the literature search, evaluated and coded articles, contributed to manuscript preparation and revision. TL conceived of the presented idea, conducted the literature search, evaluated and coded articles, contributed to manuscript preparation and revision. NG conceived of the presented idea, evaluated and coded articles, contributed to manuscript preparation and revision. IF conceived of the presented idea, contributed to manuscript preparation and revision. EC conceived of the presented idea, contributed to manuscript preparation and revision.

\section{REFERENCES}

Abell, J. W., Steenbergh, T. A., \& Boivin, M. J. (2006). Cyberporn use in the context of religiosity. Journal of Psychology and Theology, 34(2), 165-171. https://doi.org/10.1177/ 009164710603400206.

Antons, S., \& Brand, M. (2021). Diagnostic and classification considerations related to compulsive sexual behavior disorder and problematic pornography use. Current Addiction Reports, 8(3), 452-457. https://doi.org/10.1007/s40429-021-00383-7.

Benson, P. L., Roehlkepartain, E. C., \& Rude, S. P. (2003). Spiritual development in childhood and adolescence: Toward a field of inquiry. Applied Developmental Science, 7(3), 205-213. https:// doi.org/10.1207/S1532480XADS0703_12.

Blaine, B., \& Crocker, J. (1995). Religious belief salience measure. https://doi.org/10.1037/t16924-000.

Borgogna, N. C., Isacco, A., \& McDermott, R. C. (2020). A closer examination of the relationship between religiosity and problematic pornography viewing in heterosexual men. Sexual Addiction and Compulsivity, 27(1-2), 23-44. https://doi.org/10. 1080/10720162.2020.1751361.

Bradley, D. F., Grubbs, J. B., Uzdavines, A., Exline, J. J., \& Pargament, K. I. (2016). Perceived addiction to internet pornography among religious believers and nonbelievers. Sexual Addiction and Compulsivity, 23(2-3), 225-243. https://doi.org/10.1080/ 10720162.2016.1162237.

Carvalho, J., Štulhofer, A., Vieira, A. L., \& Jurin, T. (2015). Hypersexuality and high sexual desire: Exploring the structure of problematic sexuality. Journal of Sexual Medicine, 12(6), 13561367. https://doi.org/10.1111/jsm.12865.

Coleman, E., Dickenson, J. A., Girard, A., Rider, G. N., CandelarioPérez, L. E., Becker-Warner, R., .. Munns, R. (2018). An integrative biopsychosocial and sex positive model of understanding and treatment of impulsive/compulsive sexual behavior. Sexual Addiction and Compulsivity, 25(2-3), 125-152. https://doi.org/10.1080/10720162.2018.1515050.

Coleman, E., Horvath, K. J., Miner, M., Ross, M. W., Oakes, M., \& Rosser, B. R. S. (2010). Compulsive sexual behavior and risk for unsafe sex among internet using men who have sex with men. Archives of Sexual Behavior, 39(5), 1045-1053. https://doi.org/ 10.1007/s10508-009-9507-5.

Collins, G., \& Adleman, A. (2011). Breaking the cycle: Free yourself from sex addiction, porn obsession, and shame. New Harbinger.

De Jong, D. C., \& Cook, C. (2021). Roles of religiosity, obsessivecompulsive symptoms, scrupulosity, and shame in selfperceived pornography addiction: A preregistered study. Archives of Sexual Behavior, 50(2), 695-709. http://doi.org/10. 1007/s10508-020-01878-6.

Dhuffar, M. K., \& Griffiths, M. D. (2014). Understanding the role of shame and its consequences in female hypersexual behaviours: A pilot study. Journal of Behavioral Addictions, 3(4), 231-237. https://doi.org/10.1556/JBA.3.2014.4.4.

Efrati, Y. (2018a). Adolescent compulsive sexual behavior: Is it a unique psychological phenomenon? Journal of Sex and Marriage Therapy, 44(7), 687-700. https://doi.org/10.1080/ $0092623 X .2018 .1452088$.

Efrati, Y. (2018b). Adolescents with a disposition toward compulsive sexual behavior: The role of shame in willingness to seek help and treatment. Sexual Addiction and Compulsivity, 25(1), 28-45. https://doi.org/10.1080/10720162.2018.1454371.

Efrati, Y. (2019). God, I can't stop thinking about sex! the rebound effect in unsuccessful suppression of sexual thoughts among religious adolescents. Journal of Sex Research, 56(2), 146-155. https://doi.org/10.1080/00224499.2018.1461796.

Efrati, Y., \& Amichai-Hamburger, Y. (2021). Adolescents who solely engage in online sexual experiences are at higher risk for 
compulsive sexual behavior. Addictive Behaviors, 118, 106874. https://doi.org/10.1016/j.addbeh.2021.106874.

Efrati, Y., \& Mikulancer, M. (2018). Individual-based compulsive sexual behavior scale: Its development and importance in examining compulsive sexual behavior. Journal of Sex and Marital Therapy, 44(3), 249-259. https://doi.org/10.1080/ $0092623 X .2017 .1405297$.

Ellison, C. W. (1983). Spiritual well-being: Conceptualization and measurement. Journal of Psychology and Theology, 11(4), 330340. https://doi.org/10.1177/009164718301100406.

Exline, J. J., Pargament, K. I., Grubbs, J. B., \& Yali, A. M. (2014). The religious and spiritual struggles scale: Development and initial validation. Psychology of Religion and Spirituality, 6(3), 208-222. https://doi.org/10.1037/a0036465.

Exline, J. J., Yali, A. M., \& Sanderson, W. C. (2000). Guilt, discord, and alienation: The role of religious strain in depression and suicidality. Journal of Clinical Psychology, 56(12), 1481-1496. https://doi.org/ 10.1002/1097-4679(200012)56:12<1481::AID-1>3.0.CO;2-A.

Giordano, A. L., Cashwell, C. S., Lankford, C., King, K., \& Henson, R. K. (2017). Collegiate sexual addiction: Exploring religious coping and attachment. Journal of Counseling and Development, 95(2), 135-144. https://doi.org/10.1002/jcad.12126.

Giordano, A. L., \& Cecil, A. L. (2014). Religious coping, spirituality, and hypersexual behavior among college students. Sexual Addiction and Compulsivity, 21(3), 225-239. https://doi.org/10. 1080/10720162.2014.936542.

Gola, M., Lewczuk, K., \& Skorko, M. (2016). What matters: Quantity or quality of pornography use? Psychological and behavioral factors of seeking treatment for problematic pornography use. Journal of Sexual Medicine, 13(5), 815-824. https://doi.org/10.1016/j.jsxm.2016.02.169.

Griffin, B. J., Worthington, E. L., Jr., Leach, J. D., Hook, J. N., Grubbs, J., Exline, J. J., \& Davis, D. E. (2016). Sexual congruence moderates the associations of hypersexual behavior with spiritual struggle and sexual self-concept. Sexual Addiction and Compulsivity, 23(2-3), 279-295. https://doi.org/10.1080/ 10720162.2016.1150924.

Grubbs, J. B., Exline, J. J., Pargament, K. I., Hook, J. N., \& Carlisle, R. D. (2015). Transgression as addiction: Religiosity and moral disapproval as predictors of perceived addiction to pornography. Archives of Sexual Behavior, 44(1), 125-136. https://doi. org/10.1007/s10508-013-0257-z.

Grubbs, J. B., Exline, J. J., Pargament, K. I., Volk, F., \& Lindberg, M. J. (2017). Internet pornography use, perceived addiction, and religious/spiritual struggles. Archives of Sexual Behavior, 46(6), 1733-1745. https://doi.org/10.1007/s10508-016-0772-9.

Grubbs, J. B., Grant, J. T., \& Engelman, J. (2018). Self-identification as a pornography addict: Examining the roles of pornography use, religiousness, and moral incongruence. Sexual Addiction and Compulsivity, 25(4), 269-292. https://doi.org/10.1080/ 10720162.2019.1565848.

Grubbs, J. B., Hoagland, C. K., Lee, B. N., Grant, J. T., Davison, P., Reid, R. C., \& Kraus, S. W. (2020). Sexual addiction 25 years on: A systematic and methodological review of empirical literature and an agenda for future research. Clinical Psychology Review, 82, 101925. https://doi.org/10.1016/j.cpr.2020.101925.

Grubbs, J. B., Kraus, S. W., \& Perry, S. L. (2019). Self-reported addiction to pornography in a nationally representative sample:
The roles of use habits, religiousness, and moral incongruence. Journal of Behavioral Addictions, 8(1), 88-93. https://doi.org/ 10.1556/2006.7.2018.134.

Grubbs, J. B., Kraus, S. W., Perry, S. L., Lewczuk, K., \& Gola, M. (2020). Moral incongruence and compulsive sexual behavior: Results from cross-sectional interactions and parallel growth curve analyses. Journal of Abnormal Psychology, 129(3), 266278. https://doi.org/10.1037/abn0000501.

Grubbs, J. B., Lee, B. N., Hoagland, K., Kraus, S. W., \& Perry, S. L. (2020). Addiction or transgression? Moral incongruence and self-reported problematic pornography use in a nationally representative sample. Clinical Psychological Science, 8(5), 936946. https://doi.org/10.1177/2167702620922966.

Grubbs, J. B., Perry, S. L., Wilt, J. A., \& Reid, R. C. (2019). Pornography problems due to moral incongruence: An integrative model with a systematic review and meta-analysis. Archives of Sexual Behavior, 48(2), 397-415. https://doi.org/10. 1007/s10508-018-1248-x.

Grubbs, J. B., Volk, F., Exline, J. J., \& Pargament, K. I. (2015). Internet pornography use: Perceived addiction, psychological distress, and the validation of a brief measure. Journal of Sex and Marital Therapy, 41(1), 83-106. https://doi.org/10.1080/ 0092623X.2013.842192.

Grubbs, J. B., Wilt, J. A., Exline, J. J., \& Pargament, K. I. (2018). Predicting pornography use over time: Does self-reported "addiction" matter? Addictive Behaviors, 82, 57-64. https://doi. org/10.1016/j.addbeh.2018.02.028.

Grubbs, J. B., Wilt, J. A., Exline, J. J., Pargament, K. I., \& Kraus, S. W. (2018). Moral disapproval and perceived addiction to internet pornography: A longitudinal examination. Addiction, 113(3), 496-506. https://doi.org/10.1111/add.14007.

Hall, P. (2011). A biopsychosocial view of addiction. Sexual and Relationship Therapy, 26(3), 217-228. https://doi.org/10.1080/ 14681994.2011.628310.

Hook, J. N., Farrell, J. E., Davis, D. E., Van Tongeren, D. R., Griffin, B. J., Grubbs, J. B., ... Bedics, J. D. (2015). Self-forgiveness and hypersexual behavior. Sexual Addiction and Compulsivity, 22(1), 59-70. https://doi.org/10.1080/10720162.2014.1001542.

Hook, J. N., Farrell, J. E., Ramos, M. J., Davis, D. E., Karaga, S., Van Tongeren, D. R., \& Grubbs, J. B. (2015). Religiousness and congruence between sexual values and behavior. Journal of Psychology and Christianity, 34(2), 179-188. https:/go.gale. $\mathrm{com} / \mathrm{ps} / \mathrm{i} . \mathrm{do} ? \mathrm{p}=\mathrm{AONE} \& \mathrm{u}=\mathrm{anon} \sim 67101318 \& \mathrm{id}=\mathrm{GALE} \mid \mathrm{A} 42$ $6900894 \& v=2.1 \& i t=r \& s i d=$ googleScholar\&asid $=c 6 a 7 f 847$.

Hotchkiss, J. T. (2021). The relationship between sexual compulsivity, emotional and spiritual distress of religious and non-religious internet pornography users. Journal of Religion and Health, 60(3), 1630-1651. https://doi.org/10.1007/s10943-020-01152-y.

Howden, J. (1992). Development and psychometric characteristics of the spirituality assessment scale [Doctoral dissertation]. Texas Women's University. https://www.proquest.com/dissertationstheses/development-psychometric-characteristics/docview/ 304043929/se-2? accountid $=3611$.

Iannello, N. M., Hardy, S. A., Musso, P., Lo Coco, A., \& Inguglia, C. (2019). Spirituality and ethnocultural empathy among Italian adolescents: The mediating role of religious identity formation processes. Psychology of Religion and Spirituality, 11(1), 32-41. https://doi.org/10.1037/rel0000155. 
Joseph, S., \& Diduca, D. (2007). The dimensions of religiosity scale: 20-item self-report measure of religious preoccupation, guidance, conviction, and emotional involvement. Mental Health, Religion and Culture, 10(6), 603-608. https://doi.org/10.1080/ 13674670601050295.

Kafka, M. P. (2014). What happened to hypersexual disorder? Archives of Sexual Behavior, 43(7), 1259-1261. https://doi.org/ 10.1007/s10508-014-0326-y.

Karaga, S., Davis, D. E., Choe, E., \& Hook, J. N. (2016). Hypersexuality and religion/spirituality: A qualitative review. Sexual Addiction and Compulsivity, 23(2-3), 167-181. http://dx.doi. org/10.1080/10720162.2016.1144116.

King, P. E., \& Boyatzis, C. (2015). Religious and spiritual development. In M. E. Lamb, \& R. M. Lerner (Eds.), Handbook of child psychology and developmental science: Socioemotional processes (7th ed., Vol. 3, pp. 975-1021). John Wiley and Sons.

Klein, V., Savaş, Ö., \& Conley, T. D. (2021). How WEIRD and androcentric is sex research? Global inequities in study populations. The Journal of Sex Research, 1-8. https://doi.org/10. 1080/00224499.2021.1918050.

Kohut, T., \& Štulhofer, A. (2018). The role of religiosity in adolescents' compulsive pornography use: A longitudinal assessment. Journal of Sex and Marital Therapy, 44(8), 759-775. https://doi.org/10.1080/0092623X.2018.1466012.

Kraus, S. W., Krueger, R. B., Briken, P., First, M. B., Stein, D. J., Kaplan, M. S., \& AtallaReed, E. G. M. (2018). Compulsive sexual behaviour disorder in the ICD-11. World Psychiatry, 17(1), 109-110. https://doi.org/10.1002/wps.20499.

Kraus, S. W., \& Sweeney, P. J. (2019). Hitting the target: Considerations for differential diagnosis when treating individuals for problematic use of pornography. Archives of Sexual Behavior, 48(2), 431-435. https://doi.org/10.1007/s10508-018-1301-9.

Kraus, S. W., Voon, V., \& Potenza, M. N. (2016). Should compulsive sexual behavior be considered an addiction? Addiction, 111(12), 2097-2106. https://doi.org/10.1111/add. 13297.

Leonhardt, N. D., Busby, D. M., \& Willoughby, B. J. (2021). Do you feel in control? Sexual desire, sexual passion expression, and associations with perceived compulsivity to pornography and pornography use frequency. Sexuality Research and Social Policy, 18(2), 377-389. https://doi.org/10.1007/s13178-02000465-7.

Leonhardt, N. D., Willoughby, B. J., \& Young-Petersen, B. (2018). Damaged goods: Perception of pornography addiction as a mediator between religiosity and relationship anxiety surrounding pornography use. Journal of Sex Research, 55(3), 357368. https://doi.org/10.1080/00224499.2017.1295013.

Levert, N. P. (2007). A comparison of Christian and non-Christian males, authoritarianism, and their relationship to internet pornography addiction/compulsion. Sexual Addiction and Compulsivity, 14(2), 145-166. https://doi.org/10.1080/ 10720160701354771.

Lewczuk, K., Glica, A., Nowakowska, I., Gola, M., \& Grubbs, J. B. (2020). Evaluating pornography problems due to moral incongruence model. The Journal of Sexual Medicine, 17(2), 300-311. https://doi.org/10.1016/j.jsxm.2019.11.259.

Lewczuk, K., Nowakowska, I., Lewandowska, K., Potenza, M. N., \& Gola, M. (2021). Frequency of use, moral incongruence and religiosity and their relationships with self-perceived addiction to pornography, internet use, social networking and online gaming. Addiction, 116(4), 889-899. https://doi.org/10.1111/ add. 15272 .

Lewczuk, K., Szmyd, J., Skorko, M., \& Gola, M. (2017). Treatment seeking for problematic pornography use among women. Journal of Behavioral Addictions, 6(4), 445-456. https://doi.org/ 10.1556/2006.6.2017.063.

Lipka, M., \& Gecewicz, C. (2017). More Americans now say they're spiritual but not religious. Pew Research Center. http://pewrsr. $\mathrm{ch} / 2 \mathrm{xP} 0 \mathrm{Y} 8 \mathrm{w}$.

Maddock, M. E., Steele, K., Esplin, C. R., Hatch, S. G., \& Braithwaite, S. R. (2019). What is the relationship among religiosity, selfperceived problematic pornography use, and depression over time? Sexual Addiction and Compulsivity, 26(3-4), 211-238. https://doi.org/10.1080/10720162.2019.1645061.

Moher, D., Liberati, A., Tetzlaff, J., \& Altman, D. G. (2009). Preferred reporting items for systematic reviews and meta-analyses: The PRISMA statement. Plos Medicine, 6(7), e1000097. https://doi-org/10.7326/0003-4819-151-4-200908180-00135.

Pargament, K. I. (1997). The psychology of religion and coping: Theory, research, practice. New York, NY: Guilford Press.

Perry, S. L. (2019). Where does masturbation fit in all this? We need to incorporate measures of solo-masturbation in models connecting sexual media use to sexual quality (or anything else). Archives of Sexual Behavior, 48(8), 2265-2269. https://doi. org/10.1007/s10508-018-1379-0.

Prause, N. (2019). Porn is for masturbation. Archives of Sexual Behavior, 48(8), 2271-2277. https://doi.org/10.1007/s10508019-1397-6.

Reid, R. C., Carpenter, B. N., \& Hook, J. N. (2016). Investigating correlates of hypersexual behavior in religious patients. Sexual Addiction and Compulsivity, 23(2-3), 296-312. https://doi.org/ 10.1080/10720162.2015.1130002.

Reid, R., Carpenter, B. N., Hook, J. N., Garos, S., Manning, J. C., Gilliland, R., ... Fong, T. (2012). Report of findings in a DSM-5 field trial for hypersexual disorder. Journal of Sexual Medicine, 9(11), 2868-2877. https://doi.org/10.1111/j.1743-6109.2012. 02936.x.

Reid, R., Garos, S., \& Carpenter, B. N. (2011). Reliability, validity, and psychometric development of the hypersexual behavior inventory in an outpatient sample of men. Sexual Addiction and Compulsivity, 18(1), 30-51. https://doi.org/10.1080/10720162. 2011.555709.

Rosmarin, D. H., \& Pirutinsky, S. (2019). Problematic sexual behavior and religion among adult Jewish males: An initial study. American Journal of Men's Health, 13(1), 9. https://doi. org/10.1177/1557988318823586.

Ross, M. W., Månsson, S., \& Daneback, K. (2012). Prevalence, severity, and correlates of problematic sexual internet use in Swedish men and women. Archives of Sexual Behavior, 41(2), 459-466. https://doi.org/10.1007/s10508-011-9762-0.

Rousseau, A., Bőthe, B., \& Štulhofer, A. (2021). Theoretical antecedents of male adolescents' problematic pornography use: A longitudinal assessment. The Journal of Sex Research, 58(3), 331-341. https://doi.org/10.1080/00224499.2020.1815637.

Salmerón-Sánchez, P., Ballester-Arnal, R., Gil-Llario, M., \& MorellMengual, V. (2017). Sexual compulsivity and sexual sensation 
seeking: A preliminary approach among male sex workers compared to gay men in Spain. Journal of Sex and Marital Therapy, 43(1), 56-67. https://doi.org/10.1080/0092623X.2015. 1114547.

Skegg, K., Nada-Raja, S., Dickson, N., \& Paul, C. (2010). Perceived "out of control" sexual behavior in a cohort of young adults from the Dunedin Multidisciplinary Health and development study. Archives of Sexual Behavior, 39(4), 968-978. https://doi. org/10.1007/s10508-009-9504-8.

Štulhofer, A., Jurin, T., \& Briken, P. (2016). Is high sexual desire a facet of male hypersexuality? Results from an online study. Journal of Sex and Marital Therapy, 42(8), 665-680. https://doi. org/10.1080/0092623X.2015.1113585.

Thompson, M. P., \& Morrison, D. J. (2013). Prospective predictors of technology-based sexual coercion by college males. Psychology of Violence, 3(3), 233-246. https://doi.org/10.1037/a0030904.

Timberlake, D., Meyer, D., Hitchings, S., Oakley, A., Stoltzfus, L., Aguirre, S., \& Plumb, A. (2016). Sexually compulsive behaviors: Implications for attachment, early life stressors, and religiosity. Sexual Addiction and Compulsivity, 23(4), 361-373. https://doi. org/10.1080/10720162.2016.1189862.

Volk, F., Thomas, J., Sosin, L., Jacob, V., \& Moen, C. (2016). Religiosity, developmental context, and sexual shame in pornography users: A serial mediation model. Sexual Addiction and Compulsivity, 23(2-3), 244-259. https://doi.org/10.1080/ 10720162.2016.1151391.

Wilt, J. A., Cooper, E. B., Grubbs, J. B., Exline, J. J., \& Pargament, K. I. (2016). Associations of perceived addiction to internet pornography with religious/spiritual and psychological functioning. Sexual Addiction and Compulsivity, 23(2-3), 260-278. https://doi.org/10.1080/10720162.2016. 1140604 .

World Health Organization (2018). International classification of diseases for mortality and morbidity statistics (11th Revision). Retrieved from https://icd.who.int/browse11/l-m/en.

Zilberman, N., Yadid, G., Efrati, Y., Neumark, Y., \& Rassovsky, Y. (2018). Personality profiles of substance and behavioral addictions. Addictive Behaviors, 82, 174-181. https://doi.org/10.1016/ j.addbeh.2018.03.007.

Zimmer, F., \& Imhoff, R. (2020). Abstinence from masturbation and hypersexuality. Archives of Sexual Behavior, 49(4), 13331343. https://doi.org/10.1007/s10508-019-01623-8.

Zinnbauer, B. J., Pargament, K. I., \& Scott, A. B. (1999). The emerging meanings of religiousness and spirituality: Problems and prospects. Journal of Personality, 67(6), 889-919. https:// doi.org/10.1111/1467-6494.00077. 